TRANSACTIONS OF THE

AMERICAN MATHEMATICAL SOCIETY

Volume 363, Number 5, May 2011, Pages 2357-2391

S 0002-9947(2010)05046-9

Article electronically published on November 19, 2010

\title{
ON ASYMPTOTIC STABILITY OF GROUND STATES OF NLS WITH A FINITE BANDS PERIODIC POTENTIAL IN 1D
}

\author{
SCIPIO CUCCAGNA AND NICOLA VISCIGLIA
}

AbStRACT. We consider a nonlinear Schrödinger equation

$$
i u_{t}-h_{0} u+\beta\left(|u|^{2}\right) u=0,(t, x) \in \mathbb{R} \times \mathbb{R},
$$

with $h_{0}=-\frac{d^{2}}{d x^{2}}+P(x)$ a Schrödinger operator with finitely many spectral bands. We assume the existence of an orbitally stable family of ground states. Exploiting dispersive estimates in Cuccagna (2008), Cuccagna and Visciglia (2009), and following the argument in Cuccagna (to appear) we prove that under appropriate hypotheses the ground states are asymptotically stable.

\section{INTRODUCTION}

Consider $h_{0}=-\frac{d^{2}}{d x^{2}}+P(x)$, with $P(x) \equiv P(x+1)$ a smooth periodic potential of period 1 in $x \in \mathbb{R}$, and consider the NLS

$$
i u_{t}-h_{0} u+\beta\left(|u|^{2}\right) u=0,(t, x) \in \mathbb{R} \times \mathbb{R} .
$$

In this paper we study the asymptotic stability of standing waves $u(t, x)=e^{i t \omega} \phi_{\omega}(x)$ with $\phi_{\omega}(x)>0$ for all $x$. We consider the following hypotheses.

(H1) The spectrum $\sigma\left(h_{0}\right)$ is formed by finitely many bands. We choose $h_{0}$ so that $\inf \sigma\left(h_{0}\right)=0$.

(H2) $\beta(0)=\beta^{\prime}(0)=\beta^{\prime \prime}(0)=0, \beta \in C^{\infty}(\mathbb{R}, \mathbb{R})$.

(H3) There exists a $p \in(1, \infty)$ such that for every $k=0,1$,

$$
\left|\frac{d^{k}}{d v^{k}} \beta\left(v^{2}\right)\right| \lesssim|v|^{p-k-1} \quad \text { if }|v| \geq 1
$$

(H4) There exists an open interval $\mathcal{O} \subseteq(0,+\infty)$ such that $h_{0} u-\beta\left(|u|^{2}\right) u=-\omega u$ admits a $C^{1}$-family of ground states $\phi_{\omega}(x)$ for $\omega \in \mathcal{O}$.

(H5) $\frac{d}{d \omega}\left\|\phi_{\omega}\right\|_{L^{2}(\mathbb{R})}^{2}>0$ for $\omega \in \mathcal{O}$.

(H6) Set $L_{+}=h_{0}+\omega-\beta\left(\phi_{\omega}^{2}\right)-2 \phi_{\omega}^{2} \beta^{\prime}\left(\phi_{\omega}^{2}\right)$. Then $\operatorname{ker}\left(L_{+}\right)=0$ and $L_{+}$has exactly one negative eigenvalue, which is simple.

By ShS the map $\omega \rightarrow \phi_{\omega} \in H^{1}(\mathbb{R})$ is $C^{2}$, and by We1, GSS (H5) yields orbital stability of the ground state $e^{i \omega t} \phi_{\omega}(x)$. Here we investigate asymptotic stability. We need some additional hypotheses. In $\S 2$ we introduce the linearization $H_{\omega}$. By standard arguments $\sigma_{e}\left(H_{\omega}\right)=\bigcup_{ \pm} \pm\left(\omega+\sigma\left(h_{0}\right)\right)$. In particular $\sigma_{e}\left(H_{\omega}\right)$ is a, finite by (H1), union of intervals. We call thresholds of $H_{\omega}$ the numbers in $\mathbb{R}$ which are extremes of these intervals.

Received by the editors September 27, 2008.

2010 Mathematics Subject Classification. Primary 35Q55.

(C)2010 American Mathematical Society Reverts to public domain 28 years from publication 
(H7) Let $H_{\omega}$ be the linearized operator around $e^{i t \omega} \phi_{\omega}$; see Section 2. Then $H_{\omega}$ has a certain number of simple positive eigenvalues that for any $j$ : $\lambda_{j}(\omega) \notin \sigma_{e}\left(H_{\omega}\right) ; 2 \lambda_{j}(\omega) \in \sigma_{e}\left(H_{\omega}\right)$ and is not a threshold. $H_{\omega}$ does not have other eigenvalues, and each threshold of $H_{\omega}$ is not a resonance for $H_{\omega}$.

(H8) For multi-indexes $m=\left(m_{1}, m_{2}, \ldots\right)$ and $n=\left(n_{1}, \ldots\right)$, setting $\lambda(\omega)=$ $\left(\lambda_{1}(\omega), \ldots\right)$ and $(m-n) \cdot \lambda=\sum\left(m_{j}-n_{j}\right) \lambda_{j}$, we have the following two nonresonance hypotheses:

(i) $(m-n) \cdot \lambda(\omega)=0$ implies $m=n$ if $|m| \leq 3$ and $|n| \leq 3$;

(ii) $(m-n) \cdot \lambda(\omega)$ is not a threshold of $H_{\omega}$ for all $(m, n)$ with $|m|+|n| \leq 3$.

(H9) We assume the nondegeneracy hypothesis, Hypothesis 4.4.

Theorem 1.1. Let $\omega_{0} \in \mathcal{O}$ and $\phi_{\omega_{0}}(x)$ be a ground state. Let $u(t, x)$ be a solution of (1.1). Assume (H1)-(H9). Then, there exist an $\epsilon_{0}>0$ and $a C>0$ such that if $\inf _{\gamma \in[0,2 \pi]}\left\|u_{0}-e^{i \gamma} \phi_{\omega}\right\|_{H^{1}(\mathbb{R})}<\epsilon<\epsilon_{0}$, then there exist $\omega_{+} \in \mathcal{O}, \theta \in C^{1}(\mathbb{R} ; \mathbb{R})$ and $u_{+} \in H^{1}(\mathbb{R})$ with $\left\|u_{+}\right\|_{H^{1}(\mathbb{R})} \leq C \epsilon$ such that

$$
\lim _{t \rightarrow \infty}\left\|u(t, \cdot)-e^{i \theta(t)} \phi_{\omega_{+}}-e^{-i t h_{0}} u_{+}\right\|_{H^{1}}=0 .
$$

Theorem 1.1 is a transposition of more general results on the NLS with $P(x) \equiv 0$ proved for 1D in [C1] and for dimensions 2 and higher in [CT], [CM]. There is now a substantial literature on asymptotic stability of ground states of the NLS, which starts with work by Soffer and Weinstein [SW1-SW2 and Buslaev and Perelman [BP1 - BP2 in the early 1990s; see also [PW], [Wd1, [SW3. In this decade we also have further work in [C4]-C5], TY1- TY3, [BS], [SW4, GNT, GS1GS2, M1 - M2]. For some results on multisolitons see [P], [RSS. We highlight in particular the breakthrough work [GS2, later refined and extended in [C1, CT, [CM]. The present paper and the above references focus on asymptotic stability of spatially localized standing waves of equation (1.1). For a discussion of linear and orbital stability of spatially periodic or antiperiodic standing waves, see $[\mathrm{BR}], \mathrm{GH}]$ and the references therein.

The framework for Theorem 1.1 is, by now, classical. After breaking canonically the solutions $u(t, x)$ into a ground state plus a reminder; see $\S 2$, we consider an appropriate generalized NLS for the reminder $R$; see (2.1). $R$ can be decomposed using an appropriate time varying frame associated to the spectral decomposition of the linearization $H_{\omega(t)}$. Even though the spectrum of $i H_{\omega(t)}$ is on the imaginary axis, the continuous spectrum can be thought of as a stable spectrum. The discrete spectrum corresponds to central directions. As a general rule the main difficulty consists in showing that the discrete modes of $R$ decay to 0 , that is, in some sense, showing stability in the central manifold. The stabilization mechanism is known in various special situations and, in our opinion, is fundamentally well understood, although there are theorems yet to be proved. In particular, in the references listed at the end of this paper, the Hamiltonian structure of the NLS is not sufficiently exploited. We refer to [CM], C6 for further discussion on this theme. In this respect, the present paper presents no novelties. With hypotheses $(\mathrm{H} 6)-(\mathrm{H} 8)$ we consider a special case where the Hamiltonian structure of the NLS is exploited, but which is already known in the literature. The novelty in this paper is that, by exploiting dispersive estimates in [C2] on $e^{i t h_{0}}$ and extending them to $e^{i t H_{\omega}}$, we are able to develop the theory of radiation in the novel context involving an NLS with a linear periodic potential. So far the literature on asymptotic stability 
has treated only cases $h_{0}=-\partial_{x}^{2}+P(x)$ with $P(x)$ either 0 or a short range potential, except for the special case on Lamé potentials in [C3]. We generalize [C3] considerably, in particular by considering finite energy solutions in (1.1) without imposing further decay conditions at infinity for the initial datum $u_{0}(x)$ and also by easing considerably the hypotheses on $\beta\left(|u|^{2}\right)$. Furthermore, [C3 treats only 2 band potentials, while in this paper $h_{0}$ can have any finite number of bands. To prove Theorem 1.1 we need to use [M1]-M2] as smoothing estimates; see also the elaborations [C1], [CT]. To prove Stricharz estimates with Birman-Solomjak rather than Lebesgue spaces, we need to adapt a well-known lemma due to Christ and Kiselev [CK]; see Lemma 3.1 of [SmS].

It would be interesting to consider the case when $\sigma\left(h_{0}\right)$ has infinitely many bands, but in that case $H_{\omega}$ can have infinitely many eigenvalues, a situation we avoid by assuming that $\sigma\left(h_{0}\right)$ has finitely many bands. Theorem 1.1 can be generalized (see [CM] by assuming:

$\left(\mathrm{H} 7^{\prime}\right) H_{\omega}$ has a certain number of simple positive eigenvalues such that for any $j$ there is an $N_{j} \in \mathbb{N}$ such that $\ell \lambda_{j}(\omega) \notin \sigma_{e}\left(H_{\omega}\right)$ for all $\ell \in \mathbb{Z}$ with $0 \leq \ell \leq N_{j}$ and that $\left(N_{j}+1\right) \lambda_{j}(\omega) \in \sigma_{e}\left(H_{\omega}\right)$ but is not a threshold of $H_{\omega}$. $H_{\omega}$ does not have other eigenvalues, and each threshold of $H_{\omega}$ is not a resonance. We set $N=\max _{j} N_{j}$.

$\left(\mathrm{H} 8^{\prime}\right)$ For multi-indexes $m=\left(m_{1}, m_{2}, \ldots\right)$ and $n=\left(n_{1}, \ldots\right)$, setting $\lambda(\omega)=$ $\left(\lambda_{1}(\omega), \ldots\right)$ and $(m-n) \cdot \lambda=\sum\left(m_{j}-n_{j}\right) \lambda_{j}$, we have the following two nonresonance hypotheses:

(i) $(m-n) \cdot \lambda(\omega)=0$ implies $m=n$ if $|m| \leq N+2$ and $|n| \leq N+2$;

(ii) $(m-n) \cdot \lambda(\omega)$ is not a threshold of $H_{\omega}$ for all $(m, n)$ with $|m|+|n| \leq$ $N+2$.

$\left(\mathrm{H} 9^{\prime}\right)$ There is an appropriate substitute of Hypothesis 4.4, and we replace Lemma 4.3 with an appropriate hypothesis; see Hypothesis 4.4 of [C6].

We remark that [C1] revises [C7] but is quite different from [C7]. In [C7] Lemma 5.4 is wrong. Notice that the material in $\S 5$ of [C7] can be saved proceeding as in the present paper. To shorten the paper a bit we skip some proofs which are variations of proofs already in the literature. All details can be found in [CV2], which is an extended version of the present paper.

Notation. For $s \in \mathbb{R},\|u\|_{L_{x}^{p, s}}:=\left\|\langle x\rangle^{s} u\right\|_{L_{x}^{p}}$. In particular $L_{x}^{p}=L_{x}^{p, 0}$. We set $\langle f, g\rangle=\int_{\mathbb{R}}{ }^{t} f(x) \overline{g(x)} d x$, with $f(x)$ and $g(x)$ column vectors, ${ }^{t} A$ the transpose and $\bar{g}$ the complex conjugate of $g$. Sometimes we write $\langle f, g\rangle_{x}$ or $\langle f, g\rangle_{t}$ to emphasize the variable of integration. We write $\langle f, g\rangle_{s x}=\int_{\mathbb{R}^{2}}{ }^{t} f(s, x) \overline{g(s, x)} d s d x$. Given $x \in \mathbb{R}$ set $x^{+}=x \vee 0$ and $x^{-}=(-x) \vee 0 . R_{H}(z)=(H-z)^{-1}$. $W^{k, p}(\mathbb{R})$ is the space of tempered distributions $f(x)$ such that $\left(1-\partial_{x}^{2}\right)^{k / 2} f \in L^{p}(\mathbb{R}) . \mathbb{R}^{-}=(-\infty, 0), \mathbb{R}^{+}=$ $(0, \infty)$. Given two functions $f(x)$ and $g(x)$ with values in $\mathbb{C}^{2}$, whose elements are column vectors, their Wronskian is the scalar $W[f, g](x)={ }^{t} f^{\prime}(x) g(x)-{ }^{t} f(x) g^{\prime}(x)$. A pair $(r, p)$ of numbers is said to be admissible if $(r, p) \in[4, \infty] \times[2, \infty]$ with $2 / r=1 / 2-1 / p$. We set $\vec{e}_{1}={ }^{t}(1,0)$ and $\vec{e}_{2}={ }^{t}(0,1)$. Set $\operatorname{diag}(a, b)$ for the diagonal $2 \times 2$ matrix with $(a, b)$ on the diagonal. Given a metric space $X$, with $k \in X$ and $S \subseteq X$, we denote the distance of $k$ from $S$ by $\operatorname{dist}(k, S)$. For a pair of Banach spaces $X$ and $Y$ we denote by $B(X, Y)$ the Banach space of bounded linear operators from $X$ to $Y . \mathcal{S}\left(\mathbb{R}^{n}\right)$ is the space of Schwarz functions. 


\section{Linearization, MOdulation AND SET UP}

We will use the following theorem, which follows from classical arguments We1, GS1-GS2; see also the proof in Appendix A of [C1, which can be repeated omitting translation:

Theorem 2.1. Suppose that $e^{i \omega t} \phi_{\omega}(x)$ satisfies $(\mathrm{H} 1)-(\mathrm{H} 6)$ and $\phi_{\omega}(x)>0$ for all $x$. Then $\exists \epsilon>0$ and a $A_{0}(\omega)>0$ such that for any $\left\|u(0, x)-\phi_{\omega}\right\|_{H_{x}^{1}}<\epsilon$ we have for the corresponding solution $\inf \left\{\left\|u(t, x)-e^{i \gamma} \phi_{\omega}(x)\right\|_{H_{x}^{1}}: \gamma \in \mathbb{R}\right\}<A_{0}(\omega) \epsilon$.

Setting $u(t, x)=e^{i \Theta(t)}\left(\phi_{\omega(t)}(x)+r(t, x)\right), \Theta(t)=\int_{0}^{t} \omega(s) d s+\gamma(t)$ we get

$$
\begin{aligned}
& i r_{t}=h_{0} r+\omega(t) r-\beta\left(\phi_{\omega(t)}^{2}\right) r-\beta^{\prime}\left(\phi_{\omega(t)}^{2}\right) \phi_{\omega(t)}^{2} r \\
& -\beta^{\prime}\left(\phi_{\omega(t)}^{2}\right) \phi_{\omega(t)}^{2} \bar{r}+\dot{\gamma}(t) \phi_{\omega(t)}-i \dot{\omega}(t) \partial_{\omega} \phi_{\omega(t)}+\dot{\gamma}(t) r+n(r, \bar{r})
\end{aligned}
$$

for $n(r, \bar{r})=O\left(r^{2}\right), \overline{n(r, \bar{r})}=n(\bar{r}, r)$. Set ${ }^{t} R=(r, \bar{r}),{ }^{t} \Phi_{\omega}=\left(\phi_{\omega}, \phi_{\omega}\right)$. Given $\sigma_{1}=\left[\begin{array}{ll}0 & 1 \\ 1 & 0\end{array}\right], \sigma_{2}=\left[\begin{array}{cc}0 & i \\ -i & 0\end{array}\right], \sigma_{3}=\left[\begin{array}{cc}1 & 0 \\ 0 & -1\end{array}\right]$, we write

$$
H_{\omega}=\sigma_{3}\left[h_{0}+\omega-\beta\left(\phi_{\omega}^{2}\right)-\beta^{\prime}\left(\phi_{\omega}^{2}\right) \phi_{\omega}^{2}\right]+i \sigma_{2} \beta^{\prime}\left(\phi_{\omega}^{2}\right) \phi_{\omega}^{2} .
$$

The equation (1.1) is Hamiltonian, because there is a real valued function $F\left(|u|^{2}\right)$ with $\beta\left(|u|^{2}\right) u=\partial_{\bar{u}}\left(F\left(|u|^{2}\right)\right)$ and $F(0)=0$. Then $-n(r, \bar{r})=\partial_{\bar{r}} G(R)$ with $G(R)$ real valued, and $G(0)=0$. For ${ }^{t} G^{\prime}(R)=\left(G_{r}, G_{\bar{r}}\right)$ we rewrite the above equation as

$$
i R_{t}=H_{\omega} R+\sigma_{3} \dot{\gamma} R+\sigma_{3} \dot{\gamma} \Phi-i \dot{\omega} \partial_{\omega} \Phi+\sigma_{3} \sigma_{1} G^{\prime}(R) .
$$

Set $H_{0}(\omega)=\sigma_{3}\left(h_{0}+\omega\right)$ and $V(\omega)=H_{\omega}-H_{0}(\omega)$. The essential spectrum is

$$
\sigma_{e}=\sigma_{e}\left(H_{\omega}\right)=\sigma_{e}\left(H_{0}(\omega)\right)=\bigcup_{ \pm} \pm\left(\sigma\left(h_{0}\right)+\omega\right) .
$$

Here 0 is an isolated eigenvalue. Given an operator $L$ we set $N_{g}(L)=\bigcup_{j \geq 1} N\left(L^{j}\right)$ and $N(L)=\operatorname{ker} L$. We2 implies that, if $\{\cdot\}$ means span, $N_{g}\left(H_{\omega}^{*}\right)=\left\{\Phi, \sigma_{3} \partial_{\omega} \Phi\right\}$.

For each $j$ we consider a generator $\xi_{j} \in \operatorname{ker}\left(H_{\omega}-\lambda_{j}\right)$ such that $\left\langle\xi_{j}, \sigma_{3} \xi_{j}\right\rangle=1$. We expand $R(t) \in N_{g}^{\perp}\left(H_{\omega(t)}^{*}\right)$ into

$$
R(t)=\left(z \cdot \xi+\bar{z} \cdot \sigma_{1} \xi\right)+f(t) \in\left[\sum_{j, \pm} \operatorname{ker}\left(H_{\omega(t)} \mp \lambda_{j}(\omega(t))\right)\right] \oplus L_{c}^{2}\left(H_{\omega(t)}\right) .
$$

Correspondingly we express $(2.1)$ as

$$
\begin{aligned}
i \dot{z}_{j} \xi_{j}-\lambda_{j}(\omega) z_{j} \xi_{j} & =P_{\operatorname{ker}\left(H_{\omega}-\lambda_{j}\right)}\left(\dot{\gamma}(\omega, R) \sigma_{3} R+\sigma_{3} \sigma_{1} G^{\prime}(R)\right. \\
& \left.-i z_{j} \dot{\omega}(\omega, R) \partial_{\omega} \xi_{j}+i \dot{\omega}(\omega, R) \partial_{\omega} P_{\operatorname{ker}\left(H_{\omega}-\lambda_{j}\right)} R\right) \\
i P_{c}\left(H_{\omega}\right) \dot{f}-H_{\omega} f & =P_{c}\left(H_{\omega}\right)\left(\dot{\gamma}(\omega, R) \sigma_{3} R+\sigma_{3} \sigma_{1} G^{\prime}(R)+i \dot{\omega}(\omega, R) \partial_{\omega} P_{c}\left(H_{\omega}\right) R\right) .
\end{aligned}
$$

\section{Spacetime estimates For $H_{\omega}$}

We list a number of linear estimates needed in the stability argument.

Lemma 3.1 (Dispersive estimates). There exists a projection $\pi_{\omega}: L_{x}^{2} \rightarrow L_{x}^{2}$ with $\left[\pi_{\omega}, H_{\omega}\right]=0$ such that if we set $\mathcal{U}(t)=\pi_{\omega} e^{i t H_{\omega}} P_{c}\left(H_{\omega}\right)$ and $\mathcal{V}(t) \equiv$ $\left(1-\pi_{\omega}\right) e^{i t H_{\omega}} P_{c}\left(H_{\omega}\right)$, then we have for $C(\omega)>0$ semicontinuous in $\omega$ and for all $t \in \mathbb{R}$

$$
\|\mathcal{U}(t)\|_{B\left(L_{x}^{1}, L_{x}^{\infty}\right)} \leq C\langle t\rangle^{-\frac{1}{3}} \text { and }\|\mathcal{V}(t)\|_{B\left(L_{x}^{1}, L_{x}^{\infty}\right)} \leq C|t|^{-\frac{1}{2}}
$$


The proof is sketched in $\S 9$.

For every $1 \leq p, q \leq \infty$ we introduce the Birman-Solomjak spaces

$$
\ell^{p}\left(\mathbb{Z}, L_{t}^{q}[n, n+1]\right) \equiv\left\{f \in L_{l o c}^{q}(\mathbb{R}) \text { s.t. }\left\{\|f\|_{L^{q}[n, n+1]}\right\}_{n \in \mathbb{Z}} \in \ell^{p}(\mathbb{Z})\right\},
$$

endowed with the norms

$$
\begin{aligned}
& \|f\|_{\ell^{p}\left(\mathbb{Z}, L_{t}^{q}[n, n+1]\right)}^{p} \equiv \sum_{n \in \mathbb{Z}}\|f\|_{L_{t}^{q}[n, n+1]}^{p} \quad \forall 1 \leq p<\infty \text { and } 1 \leq q \leq \infty, \\
& \|f\|_{\ell^{\infty}\left(\mathbb{Z}, L_{t}^{q}[n, n+1]\right)} \equiv \sup _{n \in \mathbb{Z}}\|f\|_{L_{t}^{q}[n, n+1]} .
\end{aligned}
$$

Then we have:

Lemma 3.2 (Strichartz estimates). There exists a constant $C=C(\omega)$ upper semicontinuous in $\omega$ such that for any $k \in[0,2]$ and for every admissible pair $(r, p)$ we have

$$
\begin{aligned}
& \|\mathcal{U}(t) f\|_{\ell^{\frac{3}{2} r}\left(\mathbb{Z}, L_{t}^{\infty}\left([n, n+1], W_{x}^{k, p}\right)\right)} \leq C\|f\|_{H_{x}^{k}}, \\
& \|\mathcal{V}(t) f\|_{L_{t}^{r}\left(\mathbb{R}, W_{x}^{k, p}\right)} \leq C\|f\|_{H_{x}^{k}} .
\end{aligned}
$$

For any two admissible pairs $\left(r_{1}, p_{1}\right),\left(r_{2}, p_{2}\right)$ we have

$$
\begin{aligned}
& \left\|\int_{0}^{t} \mathcal{U}(t-s) g(s) d s\right\|_{\ell^{\frac{3}{2} r_{1}}}\left(\mathbb{Z}, L_{t}^{\infty}\left([n, n+1], W_{x}^{k, p_{1}}\right)\right) \\
& \leq C\|g\|_{\ell^{\left(\frac{3}{2} r_{2}\right)^{\prime}}\left(\mathbb{Z}, L_{t}^{1}\left([n, n+1], W_{x}^{k, p_{2}^{\prime}}\right)\right)}, \\
& \left\|\int_{0}^{t} \mathcal{V}(t-s) g(s) d s\right\|_{L_{t}^{r_{1}}\left(\mathbb{R}, W_{x}^{k, p_{1}}\right)} \leq C\|g\|_{L_{t}^{r_{2}^{\prime}}\left(\mathbb{R}, W_{x}^{k, p_{2}^{\prime}}\right)} .
\end{aligned}
$$

Lemma 3.2 is a consequence of Lemma 3.1 and an adapted $T T^{*}$ argument; see Lemma 3.1 of [C1] and our $\S 9$ for the proof.

In $\S 7$ we prove the following Kato smoothness result:

Lemma 3.3. Fix $\tau>3 / 2$. Then:

(1) there exists $C=C(\tau, \omega)$, upper semicontinuous in $\omega$, such that for any $\varepsilon \neq 0$

$$
\left\|R_{H_{\omega}}(\lambda+i \varepsilon) P_{c}\left(H_{\omega}\right) u\right\|_{L_{\lambda}^{2} L_{x}^{2,-\tau}} \leq C\|u\|_{L_{x}^{2}}
$$

(2) for any $u \in L_{x}^{2, \tau}$ the following limits exist:

$$
\lim _{\epsilon \searrow 0} R_{H_{\omega}}(\lambda \pm i \varepsilon) u=R_{H_{\omega}}^{ \pm}(\lambda) u \text { in } C^{0}\left(\sigma_{e}\left(H_{\omega}\right), L_{x}^{2,-\tau}\right) ;
$$

(3) we have

$$
\left\|R_{H_{\omega}}^{ \pm}(\lambda) P_{c}\left(H_{\omega}\right)\right\|_{B\left(L_{x}^{2, \tau}, L_{x}^{2,-\tau}\right)}<C\langle\lambda\rangle^{-\frac{1}{2}}
$$

(4) given any $u \in L_{x}^{2, \tau}$ we have

$$
P_{c}\left(H_{\omega}\right) u=\frac{1}{2 \pi i} \int_{\sigma_{e}\left(H_{\omega}\right)}\left(R_{H_{\omega}}^{+}(\lambda)-R_{H_{\omega}}^{-}(\lambda)\right) u d \lambda .
$$

Claim (1) is proved as inequality (4) in Proposition 7.1. Claim (2) is proved as formula (12) in Proposition 7.1. Claim (3) is a consequence of the proof in Proposition 7.1. The limiting absorption principle Claim (4) is Lemma 7.6 below. 
Lemma 3.4. For any $k$ and $\tau>3 / 2, \exists C=C(\tau, k, \omega)$ upper semicontinuous in $\omega$ such that:

(a) for any $f \in \mathcal{S}(\mathbb{R})$,

$$
\left\|e^{-i t H_{\omega}} P_{c}\left(H_{\omega}\right) f\right\|_{L_{t}^{2} H_{x}^{k,-\tau}} \leq C\|f\|_{H_{x}^{k}}
$$

(b) for any $g(t, x) \in \mathcal{S}\left(\mathbb{R}^{2}\right)$,

$$
\left\|\int_{\mathbb{R}} e^{i t H_{\omega}} P_{c}\left(H_{\omega}\right) g(t, \cdot) d t\right\|_{H_{x}^{k}} \leq C\|g\|_{L_{t}^{2} H_{x}^{k, \tau}}
$$

Proof. By Proposition 7.1 below, it is enough to prove Lemma 3.4, as well as Lemmas 3.5-3.6 below, for $k=0$. (a) implies (b) by duality:

$$
\begin{aligned}
& \left|\left\langle f, \sigma_{3} \int_{\mathbb{R}} e^{i t H_{\omega}} P_{c}(\omega) g(t) d t\right\rangle_{x}\right|=\left|\left\langle\langle x\rangle^{-\tau} e^{-i t H_{\omega}} P_{c}\left(H_{\omega}\right) f, \sigma_{3}\langle x\rangle^{\tau} g\right\rangle_{t, x}\right| \\
& \leq\left\|e^{-i t H_{\omega}} P_{c}\left(H_{\omega}\right) f\right\|_{L_{t}^{2} L_{x}^{2,-\tau}}\|g\|_{L_{t}^{2} L_{x}^{2, \tau}} \leq\|f\|_{L_{x}^{2}}\|g\|_{L_{t}^{2} L_{x}^{2, \tau}} .
\end{aligned}
$$

We now prove (a) for $k=0$. Let $g(t, x) \in \mathcal{S}\left(\mathbb{R}^{2}\right)$ with $g(t)=P_{c}\left(H_{\omega}\right) g(t)$. Then

$$
\begin{aligned}
& \left\langle e^{-i t H_{\omega}} f, \sigma_{3} g\right\rangle_{t, x}=\frac{1}{\sqrt{2 \pi} i} \int_{\mathbb{R}}\left\langle\left(R_{H_{\omega}}^{+}(\lambda)-R_{H_{\omega}}^{-}(\lambda)\right) f, \sigma_{3} \overline{\widehat{g}}(\lambda)\right\rangle_{x} d \lambda \\
& =\frac{1}{\sqrt{2 \pi} i} \int_{\sigma_{e}\left(H_{\omega}\right)}\left\langle\left(R_{H_{\omega}}^{+}(\lambda)-R_{H_{\omega}}^{-}(\lambda)\right) f, \sigma_{3} \overline{\widehat{g}}(\lambda)\right\rangle_{x} d \lambda .
\end{aligned}
$$

Then from Fubini and Plancherel and by (1) of Lemma 3.3 we have

$$
\left|\left\langle e^{-i t H_{\omega}} f, \sigma_{3} g\right\rangle_{t, x}\right| \leq\left\|\left(R_{H_{\omega}}^{+}(\lambda)-R_{H_{\omega}}^{-}(\lambda)\right) f\right\|_{L_{x}^{2,-\tau} L_{\lambda}^{2}\left(\sigma_{e}\left(H_{\omega}\right)\right)}\|g\|_{L_{x}^{2, \tau} L_{t}^{2}} .
$$

We have

$$
\begin{aligned}
& \left\|\left(R_{H_{\omega}}^{+}(\lambda)-R_{H_{\omega}}^{-}(\lambda)\right) f\right\|_{\ell^{2,-\tau} L_{\lambda}^{2}\left(\sigma_{e}\left(H_{\omega}\right)\right)} \\
& =\lim _{\varepsilon \searrow 0}\left\|\left(R_{H_{\omega}}(\lambda+i \varepsilon)-R_{H_{\omega}}(\lambda-i \varepsilon)\right) f\right\|_{\ell^{2,-\tau} L_{\lambda}^{2}\left(\sigma_{e}\left(H_{\omega}\right)\right) .}
\end{aligned}
$$

It is enough to prove Lemma 3.4 for $f=P_{c}\left(H_{\omega}\right) f$. Then

$$
\left\|R_{H_{\omega}}(\lambda+i \varepsilon) f\right\|_{\ell^{2,-\tau} L_{\lambda}^{2}\left(\sigma_{e}\left(H_{\omega}\right)\right)} \leq C\|f\|_{\ell^{2}}
$$

for any $\epsilon \neq 0$ and fixed $C$ follows from inequality (4) in the proof of Proposition 8.1 below.

Lemma 3.5. For any $k \in \mathbb{N}$ and $\tau>3 / 2, \exists C=C(\tau, k, \omega)$ such that $\forall g(t, x) \in$ $\mathcal{S}\left(\mathbb{R}^{2}\right)$

$$
\left\|\int_{0}^{t} e^{-i(t-s) H_{\omega}} P_{c}\left(H_{\omega}\right) g(s, \cdot) d s\right\|_{L_{t}^{2} H_{x}^{k,-\tau}} \leq C\|g\|_{L_{t}^{2} H_{x}^{k, \tau}} .
$$

Proof. By Plancherel and Hölder inequalities and by (3) of Lemma 3.3 we have

$$
\begin{aligned}
& \quad\left\|\int_{0}^{t} e^{-i(t-s) H_{\omega}} P_{c}\left(H_{\omega}\right) g(s, \cdot) d s\right\|_{L_{t}^{2} L_{x}^{2,-\tau}} \\
& \quad \leq\left\|R_{H_{\omega}}^{+}(\lambda) P_{c}\left(H_{\omega}\right) \widehat{\chi}_{[0,+\infty) *_{\lambda}} \widehat{g}(\lambda, x)\right\|_{L_{\lambda}^{2} L_{x}^{2,-\tau}} \\
& \quad \leq\|\| R_{H_{\omega}}^{+}(\lambda) P_{c}\left(H_{\omega}\right)\left\|_{B\left(L_{x}^{2, \tau}, L_{x}^{2,-\tau}\right)}\right\| \widehat{\chi}_{[0,+\infty)} *_{\lambda} \widehat{g}(\lambda, x)\left\|_{L_{x}^{2, \tau}}\right\|_{L_{\lambda}^{2}} \\
& \leq\left\|R_{H_{\omega}}^{+}(\lambda) P_{c}\left(H_{\omega}\right)\right\|_{L_{\lambda}^{\infty}\left(\mathbb{R}, B\left(L_{x}^{2, \tau}, L_{x}^{2,-\tau}\right)\right)}\|g\|_{L_{t}^{2} L_{x}^{2, \tau}} \leq C\|g\|_{L_{t}^{2} L_{x}^{2, \tau}} .
\end{aligned}
$$


Lemma 3.6. For any $k \in \mathbb{N}$ and $\tau>3 / 2, \exists C=C(\tau, k, \omega)$ such that $\forall g(t, x) \in$ $\mathcal{S}\left(\mathbb{R}^{2}\right)$

$$
\begin{aligned}
& \left\|\int_{0}^{t} \mathcal{U}(t-s) g(s, \cdot) d s\right\|_{L_{t}^{\infty} L_{x}^{2} \cap \ell^{6}\left(\mathbb{Z}, L_{t}^{\infty}\left([n, n+1], W_{x}^{k, \infty}\right)\right)} \leq C\|g\|_{L_{t}^{2} H_{x}^{k, \tau}}, \\
& \left\|\int_{0}^{t} \mathcal{V}(t-s) g(s, \cdot) d s\right\|_{L_{t}^{\infty} L_{x}^{2} \cap L_{t}^{4} W_{x}^{k, \infty}} \leq C\|g\|_{L_{t}^{2} H_{x}^{k, \tau}}
\end{aligned}
$$

Proof. For $g(t, x) \in \mathcal{S}\left(\mathbb{R}^{2}\right)$ set

$$
T_{1} g(t)=\int_{0}^{+\infty} \mathcal{U}(t-s) g(s) d s \text { and } T_{2} g(t)=\int_{0}^{+\infty} \mathcal{V}(t-s) g(s) d s .
$$

Lemma 3.4 (b) implies $f:=\int_{0}^{+\infty} e^{i s H_{\omega}} P_{c}\left(H_{\omega}\right) g(s) d s \in L_{x}^{2}$. So in particular $\pi_{\omega} f$ and $\left(1-\pi_{\omega}\right) f$ belong to $L_{x}^{2}$. For $(r, p)$ admissible we have

$$
\begin{aligned}
& \left\|T_{1} g(t)\right\|_{\ell^{\frac{3}{2} r}\left(\mathbb{Z}, L_{t}^{\infty}\left([n, n+1], L_{x}^{p}\right)\right)} \lesssim\left\|\pi_{\omega} f\right\|_{L_{x}^{2}} \lesssim\|g\|_{L_{t}^{2} L_{x}^{2, \tau}}, \\
& \left\|T_{2} g(t)\right\|_{L_{t}^{r} L_{x}^{p}} \lesssim\left\|\left(1-\pi_{\omega}\right) f\right\|_{L_{x}^{2}} \lesssim\|g\|_{L_{t}^{2} L_{x}^{2, \tau}}
\end{aligned}
$$

We get as a direct consequence of [CK], Lemma 3.1 of [SmS]:

$$
\left\|\int_{0}^{t} \mathcal{V}(t-s) g(s) d s\right\|_{L_{t}^{r} L_{x}^{p}} \lesssim\|g\|_{L_{t}^{2} L_{x}^{2, \tau}} .
$$

Our next claim is that

$$
\left\|\int_{0}^{t} \mathcal{U}(t-s) g(s) d s\right\|_{\ell^{\frac{3}{2} r}\left(\mathbb{Z}, L_{t}^{\infty}\left([n, n+1], L_{x}^{p}\right)\right)} \lesssim\|g\|_{L_{t}^{2} L_{x}^{2, \tau}}
$$

For $(r, p)=(\infty, 2)$ this follows from [CK. The case $(r, p)=(4, \infty)$ follows from an extension to Birman-Solomjak spaces of the result of [CK], which we prove in $\S 10$.

\section{Nonlinear ARGUment FOR System (2.3)}

We use the multi-index notation $z^{m}=\prod_{j} z_{j}^{m_{j}}$. We consider the Taylor expansion

$$
\sigma_{3} \sigma_{1} G^{\prime}(R)=\sum_{|m+n|=2}^{3} R_{m, n}(\omega) z^{m} \bar{z}^{n}+\sum_{|m+n|=1} z^{m} \bar{z}^{n} A_{m, n}(\omega) f+O\left(f^{2}\right)+\cdots
$$

with $R_{m, n}(\omega, x)$ and $A_{m, n}(\omega, x)$ real vectors and matrices exponentially decreasing in $x$. We have

$$
A_{m, n}(\omega)=\frac{\sigma_{3} \sigma_{1}}{m ! n !} \partial_{z}^{m} \partial_{\bar{z}}^{n} \partial_{f} G^{\prime}(0), \quad R_{m, n}(\omega)=\frac{\sigma_{3} \sigma_{1}}{m ! n !} \partial_{z}^{m} \partial_{\bar{z}}^{n} G^{\prime}(0) .
$$

We set $\delta_{j}=\left(\delta_{j 1}, \delta_{j 2}, \ldots\right)$ with $\delta_{j k}$ the Kronecker delta. We have

$$
A_{\delta_{\ell}, 0}(\omega)=\sigma_{3} \sigma_{1} \partial_{z_{\ell}} \partial_{f} G^{\prime}(0)=\sigma_{3} \sigma_{1} \partial_{z_{\ell}} \partial_{f} G^{\prime}(0)=\sigma_{3} \sigma_{1} G^{(3)}(0)\left(\cdot, \xi_{\ell}, P_{c}\left(H_{\omega}\right)\right),
$$

where $G^{(3)}(0)$ is written as a symmetric trilinear form and where one of the vectors of the triple is $\xi_{\ell}$. We have

$$
\begin{aligned}
& P_{c}\left(H_{\omega}\right) R_{\delta_{j}+\delta_{\ell}, 0}(\omega)=\frac{\sigma_{3} \sigma_{1}}{\left(\delta_{j}+\delta_{\ell}\right) !} P_{c}\left(H_{\omega}^{*}\right) \partial_{z_{j}} \partial_{z_{\ell}} G^{\prime}(0) \\
& =\frac{\sigma_{3} \sigma_{1}}{\left(\delta_{j}+\delta_{\ell}\right) !} \partial_{z_{j}} \partial_{z_{\ell}} G^{\prime}(0) \circ P_{c}\left(H_{\omega}\right)=\frac{\sigma_{3} \sigma_{1}}{\left(\delta_{j}+\delta_{\ell}\right) !} G^{(3)}(0)\left(\xi_{j}, \xi_{\ell}, P_{c}\left(H_{\omega}\right)\right) .
\end{aligned}
$$


For $O_{l o c}\left(z^{n}\right)=\sum_{\ell} O_{l o c}\left(\left|z_{\ell}^{n}\right|\right),(2.1)$ can be expressed as

$$
\begin{aligned}
& i f_{t}=\left(H_{\omega(t)}+P_{c}\left(H_{\omega}\right) \sigma_{3} \dot{\gamma}\right) f+\sum_{|m+n|=2} z^{m} \bar{z}^{n} P_{c}\left(H_{\omega}\right) R_{m, n}(\omega) \\
& +\sum_{|m+n|=1} z^{m} \bar{z}^{n} P_{c}\left(H_{\omega}\right) A_{m, n}(\omega) f+O\left(f^{2}\right)+O_{l o c}\left(z^{3}\right)
\end{aligned}
$$

and

$$
\begin{aligned}
& i \dot{z}_{j} \xi_{j}-\lambda_{j}(\omega) z_{j} \xi_{j}=P_{\operatorname{ker}\left(H_{\omega}-\lambda_{j}\right)}\left(\sum_{|m+n|=2} z^{m} \bar{z}^{n} R_{m, n}(\omega)\right. \\
& \left.+\sum_{|m+n|=3} z^{m} \bar{z}^{n} R_{m, n}^{(1)}(\omega)+\sum_{|m+n|=1} z^{m} \bar{z}^{n} A_{m, n}(\omega) f\right)+O\left(f^{2}\right)+O_{l o c}\left(z^{4}\right)
\end{aligned}
$$

with the $A_{m, n}(\omega)$ and $R_{m, n}(\omega)$ the same as the expansion of $\sigma_{3} \sigma_{1} G^{\prime}(R)$ and with the $R_{m, n}^{(1)}(\omega)$ real and exponentially decreasing vectors. We have:

Lemma 4.1. For any $C_{1}>0, \exists \epsilon\left(C_{1}\right)>0$ and $C\left(C_{1}\right)$ such that if, for $0<\epsilon<$ $\epsilon\left(C_{1}\right)$, we have $\left\|z_{j}\right\|_{L^{\infty}(0, T)}+\left\|z_{j}\right\|_{L^{4}(0, T)}^{2} \leq C_{1} \epsilon$ for all $j$, then

$$
\|f\|_{L_{t}^{2}\left((0, T), H_{x}^{1,-2}\right)}+\|f\|_{L_{t}^{6}\left((0, T), L_{x}^{\infty}\right) \cap L_{t}^{\infty}\left((0, T), H_{x}^{1}\right)}<C\left(C_{1}\right) \epsilon .
$$

Proof. Notice that $\sum_{j}\left\|z_{j}\right\|_{L_{t}^{\infty}}+\|f\|_{L_{t}^{\infty} H_{x}^{1}}<c_{0} \epsilon$ for a fixed $c_{0}$ is a consequence of orbital stability; see Theorem 2.1 above. In this proof we set $P_{c}(\omega)=P_{c}\left(H_{\omega}\right)$. We split $P_{c}(\omega)=P_{+}(\omega)+P_{-}(\omega)$ with $P_{ \pm}(\omega)$ the spectral projections in $\mathbb{R}_{ \pm} \cap \sigma_{e}\left(H_{\omega}\right)$; see Lemma 8.13 below. We now rewrite the equation for $f$. By orbital stability, Theorem 2.1, we can fix $\omega_{0}$ such that $\left|\omega(t)-\omega_{0}\right|=O(\epsilon)$ for all $t$. Following [BP2] we set

$$
\begin{aligned}
& i f_{t}=\left\{H_{\omega_{0}}+\left(\dot{\gamma}+\omega-\omega_{0}\right)\left(P_{+}\left(\omega_{0}\right)-P_{-}\left(\omega_{0}\right)\right)\right\} P_{c}\left(\omega_{0}\right) f \\
& +O_{l o c}(\epsilon f)+O\left(f^{2}\right)+O_{l o c}\left(z^{2}\right), \\
& \text { where } O_{l o c}(\epsilon f)=\left(\dot{\gamma}+\omega-\omega_{0}\right)\left(P_{c}\left(\omega_{0}\right) \sigma_{3}-\left(P_{+}\left(\omega_{0}\right)-P_{-}\left(\omega_{0}\right)\right)\right) f \\
& +\left(V(\omega)-V\left(\omega_{0}\right)\right) f+\left(\dot{\gamma}+\omega-\omega_{0}\right)\left(P_{c}(\omega)-P_{c}\left(\omega_{0}\right)\right) \sigma_{3} f
\end{aligned}
$$

with $V(\omega)$ the localized matrix potential defined under (2.1) and where the notation $O_{l o c}(\epsilon f)$ is justified by the fact that $\omega-\omega_{0}=O(\epsilon)$. Also, for any pair $s_{1}, s_{2} \in \mathbb{R}$ there is $c_{s_{1}, s_{2}}(\omega)$ upper semicontinuous in $\omega$ (see Lemma 8.13) such that for $j=0,1$,

$$
\left\|P_{c}(\omega) \sigma_{3}-\left(P_{+}(\omega)-P_{-}(\omega)\right)\right\|_{H_{x}^{j, s_{1}} \rightarrow H_{x}^{j, s_{2}}} \leq c_{s_{1}, s_{2}}(\omega)<\infty,
$$

where the case $j=1$ follows from $j=0$ by applying $H_{\omega}$ and interpolating. We have $\|f\|_{L_{t}^{6}\left((0, T), L_{x}^{\infty}\right)} \approx\left\|P_{c}\left(\omega_{0}\right) f\right\|_{L_{t}^{6}\left((0, T), L_{x}^{\infty}\right)}$ by

$$
\begin{aligned}
& \|f\|_{L_{t}^{6}\left((0, T), L_{x}^{\infty}\right)}=\left\|P_{c}\left(\omega_{0}\right) f\right\|_{L_{t}^{6}\left((0, T), L_{x}^{\infty}\right)}+\left\|\left(P_{c}(\omega)-P_{c}\left(\omega_{0}\right)\right) f\right\|_{L_{t}^{6}\left((0, T), L_{x}^{\infty}\right)} \\
& \quad=\left\|P_{c}\left(\omega_{0}\right) f\right\|_{L_{t}^{6}\left((0, T), L_{x}^{\infty}\right)}+O(\epsilon)\|f\|_{L_{t}^{6}\left((0, T), L_{x}^{\infty}\right)} .
\end{aligned}
$$

For

$$
U_{ \pm}\left(t, t^{\prime}\right)=e^{-i\left(t-t^{\prime}\right) H_{\omega_{0}}} e^{ \pm i \int_{t^{\prime}}^{t} d \tau\left(\dot{\gamma}(\tau)+\omega(\tau)-\omega_{0}\right)} P_{ \pm}\left(\omega_{0}\right)
$$


we have

$$
P_{ \pm}\left(\omega_{0}\right) f(t)=U_{ \pm}(t, 0) f(0)+\int_{0}^{t} U_{ \pm}\left(t, t^{\prime}\right)\left(O_{l o c}(\epsilon f)+O\left(f^{2}\right)+O_{l o c}\left(z^{2}\right)\right) d t^{\prime} .
$$

We set $O_{l o c}(\epsilon f)+O\left(f^{2}\right)+O_{l o c}\left(z^{2}\right)=\mathcal{X}+O\left(f^{7}\right)$. Let $\pi=\pi_{\omega_{0}}$ be the projection in Lemmas 3.1-3.2 and $\pi_{1}=1-\pi$. By (1)-(2) of Lemma 3.2 and interpolation, we have for a fixed $c_{0}\left(\omega_{0}\right)$

$$
\begin{aligned}
& \left\|U_{ \pm}(t, 0) f(0)\right\|_{L_{t}^{6}\left((0, T), L_{x}^{\infty}\right)} \leq\left\|U_{ \pm}(t, 0) f(0)\right\|_{L_{t}^{6} L_{x}^{\infty}} \\
& \lesssim\left\|U_{ \pm}(t, 0) \pi f(0)\right\|_{\ell^{6}\left(\mathbb{Z}, L_{t}^{\infty}\left([n, n+1], L_{x}^{\infty}\right)\right)}+\left\|U_{ \pm}(t, 0) \pi_{1} f(0)\right\|_{L_{t}^{4} L_{x}^{\infty}}^{\frac{2}{3}} \\
& \times\left\|U_{ \pm}(t, 0) \pi_{1} f(0)\right\|_{L_{t}^{\infty} H_{x}^{1}}^{\frac{1}{3}} \leq c_{0}\left(\omega_{0}\right)\|f(0)\|_{H_{x}^{1}} \leq c_{0}\left(\omega_{0}\right) \epsilon .
\end{aligned}
$$

Similarly, by (3)-(4) of Lemma 3.2 and interpolation, we have

$$
\begin{aligned}
& \left\|\int_{0}^{t} U_{ \pm}\left(t, t^{\prime}\right) O\left(f^{7}\right) d t^{\prime}\right\|_{L_{t}^{6}\left((0, T), L_{x}^{\infty}\right)} \lesssim\left\|\int_{0}^{t} U_{ \pm}\left(t, t^{\prime}\right) \pi_{1} O\left(f^{7}\right) d t^{\prime}\right\|_{L_{t}^{4}\left((0, T), L_{x}^{\infty}\right)}^{\frac{2}{3}} \\
& \times\left\|\int_{0}^{t} U_{ \pm}\left(t, t^{\prime}\right) \pi_{1} O\left(f^{7}\right) d t^{\prime}\right\|_{L_{t}^{\infty}\left((0, T), H_{x}^{1}\right)}^{\frac{1}{3}} \\
& +\left\|\chi_{[0, T]} \int_{0}^{t} U_{ \pm}\left(t, t^{\prime}\right) \pi O\left(f^{7}\right) d t^{\prime}\right\|_{\ell^{6}\left(\mathbb{Z}, L_{t}^{\infty}\left([n, n+1], L_{x}^{\infty}\right)\right)} .
\end{aligned}
$$

By Lemma 3.2 both terms on the right are bounded by $\left\|O\left(f^{7}\right)\right\|_{L_{t}^{1}\left((0, T), H_{x}^{1}\right)}$. Therefore, by Lemma 3.2 and Hölder inequality we have

$$
\begin{aligned}
& \left\|\int_{0}^{t} U_{ \pm}\left(t, t^{\prime}\right) O\left(f^{7}\right) d t^{\prime}\right\|_{L_{t}^{6}\left((0, T), L_{x}^{\infty}\right)} \leq c_{0}\left\|O\left(f^{7}\right)\right\|_{L_{t}^{1}\left((0, T), H_{x}^{1}\right)} \\
& \lesssim\|f\|_{L_{t}^{6}\left((0, T), L_{x}^{\infty}\right)}^{6}\|f\|_{L_{t}^{\infty}(0, T) H_{x}^{1}} \lesssim \epsilon^{7} .
\end{aligned}
$$

In a similar fashion, by Lemma 3.6 and by (1) below we have

$$
\left\|\int_{0}^{t} U_{ \pm}\left(t, t^{\prime}\right) \mathcal{X} d t^{\prime}\right\|_{L_{t}^{6}\left((0, T), L_{x}^{\infty}\right)} \leq c_{0}(\omega)\|\mathcal{X}\|_{L_{t}^{2}\left((0, T), H_{x}^{1,2}\right)} \lesssim \epsilon^{2} .
$$

We claim that we have

$$
\|\mathcal{X}\|_{L_{t}^{2} H_{x}^{1,2}} \leq C(\omega)\left(\epsilon^{2}+\|f\|_{L_{t}^{2} H_{x}^{1,-2}}^{2}\right)
$$

Indeed $\mathcal{X}=O_{l o c}(\epsilon f)+O_{l o c}(z f)+O_{l o c}\left(f^{2}\right)+O_{l o c}\left(z^{2}\right)$ with

$$
\begin{array}{cl}
\left\|O_{l o c}(\epsilon f)\right\|_{H_{x}^{1,2} L_{t}^{2}} \lesssim \epsilon\|f\|_{L_{t}^{2} H_{x}^{1,-2}} ; & \left\|O_{l o c}(z f)\right\|_{H_{x}^{1,2} L_{t}^{2}} \lesssim\|z\|_{\infty}\|f\|_{L_{t}^{2} H_{x}^{1,-2}} ; \\
\left\|O_{l o c}\left(f^{2}\right)\right\|_{L_{t}^{2} H_{x}^{1,2}} \lesssim\|f\|_{L_{t}^{2} H_{x}^{1,-2}}^{2} ; & \left\|O_{l o c}\left(|z|^{2}\right)\right\|_{H_{x}^{1,2} L_{t}^{2}} \lesssim \epsilon\left\|z^{2}\right\|_{L_{t}^{2}} \lesssim \epsilon^{2} .
\end{array}
$$

Hence (1) is correct. Now let $f=g+h$ with

$$
\begin{aligned}
i g_{t} & =\left\{H_{\omega_{0}}+\ell(t)\left(P_{+}\left(\omega_{0}\right)-P_{-}\left(\omega_{0}\right)\right)\right\} g+\mathcal{X}, \quad g(0)=f(0), \\
i h_{t} & =\left\{H_{\omega_{0}}+\ell(t)\left(P_{+}\left(\omega_{0}\right)-P_{-}\left(\omega_{0}\right)\right)\right\} h+O\left(f^{7}\right), \quad h(0)=0 .
\end{aligned}
$$

Then, by Lemmas 3.4 and 3.5 we get for a fixed $c_{0}$

$$
\|g\|_{L_{t}^{2}\left((0, T), H_{x}^{1,-2}\right)} \lesssim c_{0}\left(C_{1}+1\right) \epsilon+O\left(\epsilon^{2}\right) \leq C\left(C_{1}\right) \epsilon .
$$


Finally, by Lemma 3.4

$$
\begin{aligned}
& \int_{0}^{T}\left\|e^{-i(t-s) H_{\omega_{0}}} e^{ \pm i \int_{s}^{t} \ell(\tau) d \tau} O\left(f^{7}\right)(s)\right\|_{L_{t}^{2}\left((0, T), H_{x}^{1,-2}\right)} \\
& \lesssim \int_{0}^{T}\left\|O\left(f^{7}\right)(s)\right\|_{H_{x}^{1}} d s \lesssim \epsilon^{7} .
\end{aligned}
$$

This yields $\|h\|_{L_{t}^{2}\left((0, T), H_{x}^{1,-2}\right)} \lesssim \epsilon^{7}$ and completes the proof of Lemma 4.1.

Having obtained Lemma 4.1, we rewrite (4.1a) in the form

$$
\begin{aligned}
& i f_{t}=\left\{H_{\omega_{0}}+\left(\dot{\gamma}+\omega-\omega_{0}\right)\left(P_{+}\left(\omega_{0}\right)-P_{-}\left(\omega_{0}\right)\right)\right\} f \\
& +\sum_{|m+n|=2} z^{m} \bar{z}^{n} P_{c}\left(\omega_{0}\right) R_{m, n}(\omega)+\sum_{|m+n|=1} z^{m} \bar{z}^{n} P_{c}\left(\omega_{0}\right) A_{m, n}(\omega) f \\
& +\left(\dot{\gamma}+\omega-\omega_{0}\right)\left(P_{c}\left(\omega_{0}\right) \sigma_{3}-\left(P_{+}\left(\omega_{0}\right)-P_{-}\left(\omega_{0}\right)\right)\right) f+\left(V(\omega)-V\left(\omega_{0}\right)\right) f \\
& +\left(\dot{\gamma}+\omega-\omega_{0}\right)\left(P_{c}(\omega)-P_{c}\left(\omega_{0}\right)\right) \sigma_{3} f+O\left(f^{2}\right)+O_{l o c}\left(z^{3}\right) \\
& +\left(P_{c}(\omega)-P_{c}\left(\omega_{0}\right)\right)\left(\sum_{|m+n|=2} z^{m} \bar{z}^{n} R_{m, n}(\omega)+\sum_{|m+n|=1} z^{m} \bar{z}^{n} A_{m, n}(\omega) f\right) .
\end{aligned}
$$

We then set

$$
f_{2}=f+\sum_{|m+n|=2} R_{H_{\omega_{0}}}^{+}\left((m-n) \cdot \lambda\left(\omega_{0}\right)\right) P_{c}\left(H_{\omega_{0}}\right) R_{m, n}(\omega) z^{m} \bar{z}^{n} .
$$

We will need below:

Lemma 4.2. Assume the hypotheses of Lemma 4.1. Then for $s>1$ sufficiently large we can decompose $f_{2}=h_{1}+h_{2}+h_{3}+h_{4}$ with:

(1) for a fixed $c_{0}\left(\omega_{0}\right),\left\|h_{1}\right\|_{L_{t}^{2} L_{x}^{2,-s}} \leq c_{0}\left(\omega_{0}\right)\|f(0)\|_{H^{1}} \leq c_{0}\left(\omega_{0}\right) \epsilon$;

(2) for a fixed $c_{1}\left(\omega_{0}\right),\left\|h_{2}\right\|_{L_{t}^{2} L_{x}^{2,-s}} \leq c_{1}\left(\omega_{0}\right)|z(0)|^{2} \leq c_{1}\left(\omega_{0}\right) \epsilon^{2}$;

(3) $\left\|h_{3}\right\|_{L_{t}^{2} L_{x}^{2,-s}}=O\left(\epsilon^{2}\right)$;

(4) $\left\|h_{4}\right\|_{L_{t}^{2} L_{x}^{2,-s}}=O\left(\epsilon^{2}\right)$.

Proof. The proof is basically that of $\S 4$ in [CM]. It uses the fact that for a constant $C=C\left(\Lambda, \omega_{0}\right)$ upper semicontinuous in $\omega_{0}$ and in $\Lambda>\omega_{0}$ we have

$$
\left\|U_{ \pm}\left(t, t^{\prime}\right) R_{H_{\omega}}^{+}(\Lambda) P_{c} g\right\|_{L_{x}^{2,-s}}<C\left\langle t-t^{\prime}\right\rangle^{-\frac{3}{2}}\|g\|_{L_{x}^{2, s}}, s>s_{0} .
$$

The weighted estimate (5) can be proved proceeding along the lines of the proof of Theorem 2.4 on pp. 135-136 of [C3] by using the estimates for the Jost functions in Lemma 9.1 and the representation in Lemma 8.12.

By substitution of (4.3) in (4.1b) we get

$$
\begin{aligned}
& i \dot{z}_{j} \xi_{j}-\lambda_{j}(\omega) z_{j} \xi_{j}=P_{\operatorname{ker}\left(H_{\omega}-\lambda_{j}\right)}\left(\sum_{|m+n|=2} z^{m} \bar{z}^{n} R_{m, n}(\omega)+\sum_{|m+n|=3} z^{m} \bar{z}^{n} R_{m, n}^{(1)}(\omega)\right. \\
& -\sum_{\left|m^{\prime}+n^{\prime}\right|=1} \sum_{|m+n|=2} z^{m+m^{\prime}} \bar{z}^{n+n^{\prime}} A_{m^{\prime}, n^{\prime}}(\omega) R_{H_{\omega_{0}}}^{+}\left((m-n) \cdot \lambda\left(\omega_{0}\right)\right) P_{c}\left(H_{\omega}\right) R_{m, n}(\omega) \\
& \left.+\sum_{|m+n|=1} z^{m} \bar{z}^{n} A_{m, n}(\omega) f_{2}+O\left(f^{2}\right)+O_{l o c}\left(z^{4}\right)\right) .
\end{aligned}
$$


Here we recall $P_{\operatorname{ker}\left(H_{\omega}-\lambda_{j}\right)}=\xi_{j}\left\langle, \sigma_{3} \xi_{j}\right\rangle$. By standard normal forms arguments there exists a change of variables $\zeta_{j}=z_{j}+\sum_{|m+n|=2}^{3} p_{j, m, n}(\omega) z^{m} \bar{z}^{n}$ with $\Im p_{j, m, n}=$ 0 for $|m+n|=2$ such that below we have $\Im a_{j, \ell}(\omega)=0$ and

$$
\begin{aligned}
& i \dot{\zeta}_{j} \xi_{j}-\lambda_{j}(\omega) \zeta_{j} \xi_{j}=\xi_{j} \sum_{\ell} a_{j, \ell}(\omega)\left|\zeta_{\ell}\right|^{2} \zeta_{j}+O_{l o c}\left(\zeta f_{2}\right)+O\left(f^{2}\right)+O_{l o c}\left(\zeta^{4}\right) \\
& -\sum_{\ell}\left|\zeta_{\ell}\right|^{2} \zeta_{j} P_{\operatorname{ker}\left(H_{\omega}-\lambda_{j}\right)} A_{0, \delta_{\ell}}(\omega) R_{H_{\omega_{0}}}^{+}\left(\lambda_{\ell}\left(\omega_{0}\right)+\lambda_{j}\left(\omega_{0}\right)\right) P_{c}\left(H_{\omega}\right) R_{\delta_{\ell}+\delta_{j}, 0}(\omega),
\end{aligned}
$$

where $O_{l o c}\left(\zeta^{n} f_{2}\right)=\sum_{\ell} O_{l o c}\left(\zeta_{\ell}^{n} f_{2}\right)$. Applying $\left\langle, \sigma_{3} \xi_{j}\right\rangle$ to (4.4) we get

$$
\begin{aligned}
& i \dot{\zeta}_{j}-\lambda_{j}(\omega) \zeta_{j}=\sum_{\ell} a_{j, \ell}(\omega)\left|\zeta_{\ell}\right|^{2} \zeta_{j}+\left\langle O_{l o c}\left(\zeta f_{2}\right)+O\left(f^{2}\right)+O_{l o c}\left(\zeta^{4}\right), \sigma_{3} \xi_{j}\right\rangle \\
& -\sum_{\ell}\left|\zeta_{\ell}\right|^{2} \zeta_{j}\left\langle A_{0, \delta_{\ell}}(\omega) R_{H_{\omega_{0}}}^{+}\left(\lambda_{\ell}\left(\omega_{0}\right)+\lambda_{j}\left(\omega_{0}\right)\right) P_{c}\left(H_{\omega}\right) R_{\delta_{\ell}+\delta_{j}, 0}(\omega), \sigma_{3} \xi_{j}\right\rangle .
\end{aligned}
$$

Recall that $\Im a_{j, \ell}(\omega)=0$. Then multiplying (4.5) by $\bar{\zeta}_{j}$ we get

$$
\begin{aligned}
& \frac{1}{2} \frac{d}{d t}\left|\zeta_{j}\right|^{2}=-\sum_{\ell}\left(\Gamma_{j, \ell}\left(\omega_{0}\right)+o(1)\right)\left|\zeta_{\ell}\right|^{2}\left|\zeta_{j}\right|^{2} \\
& +\Im\left[\overline{\zeta_{j}}\left\langle O_{l o c}\left(\zeta f_{2}\right)+O\left(f^{2}\right)+O_{l o c}\left(\zeta^{4}\right), \sigma_{3} \xi_{j}\right\rangle\right],
\end{aligned}
$$

where we use $\omega-\omega_{0}=O(\epsilon)$,

$$
\Gamma_{j, \ell}(\omega)=\Im\left\langle A_{0, \delta_{\ell}}(\omega) R_{H_{\omega}}^{+}\left(\lambda_{\ell}(\omega)+\lambda_{j}(\omega)\right) P_{c}\left(H_{\omega}\right) R_{\delta_{\ell}+\delta_{j}, 0}(\omega), \sigma_{3} \xi_{j}(\omega)\right\rangle
$$

and the continuous dependence in $\omega$ of $A_{0, \delta_{\ell}}(\omega), R_{\delta_{\ell}+\delta_{j}, 0}(\omega)$ and $P_{c}\left(H_{\omega}\right)$. We have:

Lemma 4.3. We have for $h_{j, \ell}(\omega)=\sigma_{3} \sigma_{1} G^{(3)}(0)\left(\xi_{j}, \xi_{\ell}, P_{c}\left(H_{\omega}\right)\right)$

$$
\Gamma_{j, \ell}(\omega)=\frac{\pi}{\left(\delta_{j}+\delta_{\ell}\right) !}\left\langle\delta\left(H_{\omega}-\lambda_{j}-\lambda_{\ell}\right) h_{j, \ell}(\omega), \sigma_{3} h_{j, \ell}(\omega)\right\rangle \geq 0 .
$$

The proof is in Lemma 3.4 of [6]. We assume the following, which by $2 \lambda_{j}(\omega)>\omega$ for any $j$ looks like a generic condition:

Hypothesis 4.4. We suppose that $\Gamma_{j, j}(\omega)>0$ for any $j$.

Integrating (4.6) in an interval $[0, t]$ we get

$$
\begin{aligned}
& C_{0} \sum_{j} \int_{0}^{t}\left|\zeta_{j}\right|^{4}+\sum_{j}\left|\zeta_{j}(t)\right|^{2} / 2 \\
& \leq \sum_{j}\left|\zeta_{j}(0)\right|^{2} / 2+c_{1}\left(\sum_{j} \int_{0}^{t}\left|\zeta_{j}\right|^{4}\right)^{\frac{1}{2}}\left\|f_{2}\right\|_{L_{t}^{2} L_{x}^{2,-s}}+O\left(\epsilon^{3}\right) .
\end{aligned}
$$

By Lemma $4.2,\left\|f_{2}\right\|_{L_{t}^{2} L_{x}^{2,-s}} \leq c_{2} \epsilon$ for $c_{2} \approx 1$. Then for a fixed $c \approx 1$

$$
\sum_{j} \int_{0}^{t}\left|z_{j}\right|^{4}+\sum_{j}\left|z_{j}(t)\right|^{2} \leq c \epsilon^{2} .
$$

The proof that, for ${ }^{t} f(t)=(h(t), \bar{h}(t)), h(t)$ is asymptotically free for $t \rightarrow \infty$, is similar to the analogous one in $\mathrm{CM}$. Finally, by the same argument of BP2, BS, and $[\mathrm{CM}], \lim _{t \nearrow_{\infty}} \omega(t)=\omega_{+}$for some $\omega_{+}$. Hence expressing (1) in components we obtain Theorem 1.1. 


\section{REview ON BLOCh FUnCtions FOR $h_{0}$}

By hypothesis for $\sigma\left(h_{0}\right)$ there exist $A_{0}^{ \pm}=0<A_{1}^{-}<A_{1}^{+}<\ldots<A_{n_{0}}^{-}<A_{n_{0}}^{+}<\infty$ such that

$$
\sigma\left(h_{0}\right)=\bigcup_{j=0}^{n_{0}-2}\left[A_{j}^{+}, A_{j+1}^{-}\right] \cup\left[A_{n_{0}}^{+}, \infty\right) .
$$

The sets $\left(A_{j}^{-}, A_{j}^{+}\right)$for $j=1, \ldots, n_{0}$ are nonempty spectral gaps. For $j \geq 0$ we set $a_{j}^{ \pm}=\sqrt{A_{j}^{ \pm}}$. For $j \leq 0, a_{j}^{ \pm}=-a_{-j}^{ \pm}$. For any $E \in \mathbb{C} \backslash[0, \infty)$ there is a unique $k \in \mathbb{C}_{+}$, called quasimomentum, such that $h_{0} u=E u$ has a solution of the form $\widetilde{\phi}_{ \pm}(x, k)=e^{ \pm i k x} \widetilde{m}_{ \pm}(x, k)$ with $\widetilde{m}_{ \pm}(x+1, k) \equiv \widetilde{m}_{ \pm}(x, k)$ and $\widetilde{m}_{ \pm}(0, k)=1$. The correspondence $E \rightarrow k(E)$ is a conformal map from $\mathbb{C} \backslash[0, \infty)$ into a set

$$
\mathcal{K}=\mathbb{C}_{+} \backslash\left(\bigcup_{j=1}^{n_{0}}\left(\left[n(j) \pi, n(j) \pi+i h_{j}\right] \cup\left[-n(j) \pi,-n(j) \pi+i h_{j}\right]\right),\right.
$$

with $n(j)$ strictly increasing for $j=1, \ldots, n_{0}$ and $h_{j}>0$. We set $\mathcal{T}=\{ \pm \pi n(j)$ : $\left.j=1, \ldots, n_{0}\right\}$. The inverse map $E=E(k)$ extends in an even map $\mathbb{R} \backslash \mathcal{T} \rightarrow \sigma\left(h_{0}\right)$. We have

$$
E(\pi n(j) \pm 0):=\lim _{\varepsilon \searrow 0} E(n(j) \pi \pm \varepsilon)=\pi A_{j}^{ \pm} .
$$

$E(k)$ defined in $] \pi n(j), \pi n(j+1)$ [ extends continuously in $[\pi n(j), \pi n(j+1)]$. The same holds for $\left[\pi n\left(n_{0}\right), \infty\right)$. We consider the solutions $\theta(x, k)$ and $\varphi(x, k)$ of $h_{0} u=$ $E(k) u$ which satisfy the initial conditions $\varphi(0, k)=\theta^{\prime}(0, k)=0$ and $\varphi^{\prime}(0, k)=$ $\theta(0, k)=1$. Then

$$
\tilde{\phi}_{ \pm}(x, k)=\theta(x, k)+m^{ \pm}(k) \varphi(x, k) \text { with } m^{ \pm}(k)=\frac{\varphi^{\prime}(1, k)-\theta(1, k)}{2 \varphi(1, k)} \pm i \frac{\sin k}{\varphi(1, k)} .
$$

For real $k \in \mathbb{R} \backslash \mathcal{T}$ we have $\widetilde{\phi}_{ \pm}(x, k)=\overline{\widetilde{\phi}_{\mp}(x, k)}$. Now set

$$
N^{2}(k)=\int_{0}^{1} \tilde{\phi}_{+}(x, k) \tilde{\phi}_{-}(x, k) d x .
$$

Then $N^{2}(k)>0$ for $k \in \mathbb{R} \backslash \mathcal{T}$, with $N^{2}(k)$ bounded away from 0 near $\mathcal{T}$, and $N^{2}(k) \neq 0$ for all $k \in \mathcal{K}$; see for instance $\S 2$ of $[\mathrm{C} 2$. Then there is an $a>0$ such that there is a well defined holomorphic square root $N(k)$ for $k \in \mathcal{K}$ with $\Im k<a$. Notice that the restriction $\Im k<a$ is due to monodromy issues. We extend $N(k) \neq 0$ for $\Im k \geq a$ as well, possibly as a discontinuous function. Then we introduce functions $m_{ \pm}^{0}(x, k)=\tilde{m}_{ \pm}^{0}(x, k) / N(k)$ and $\phi_{ \pm}^{0}(x, k):=e^{ \pm i k x} m_{ \pm}^{0}(x, k)$. The following fact is well known.

Theorem 5.1. The functions $\phi_{ \pm}^{0}(x, k)$ are analytic in $k \in \mathbb{R} \backslash \mathcal{T}$, and for $\mathcal{F}_{h_{0}} f(k)=$ $\int_{\mathbb{R}} \phi_{+}^{0}(x, k) f(x) d x$ we have

$$
\begin{aligned}
& \int_{\mathbb{R}}|f(y)|^{2} d y=\int_{\mathbb{R}}\left|\mathcal{F}_{h_{0}} f(k)\right|^{2} d k ; \\
& f(x)=\int_{\mathbb{R}} \phi_{-}^{0}(x, k) \mathcal{F}_{h_{0}} f(k) d k ; \\
& \mathcal{F}_{h_{0}}\left(h_{0} f\right)(k)=E(k) \mathcal{F}_{h_{0}} f(k) \text { for } k \in \mathbb{R} \backslash \mathcal{T} ; \\
& e^{i t h_{0}}(x, y)=\int_{\mathbb{R}} e^{i(t E(k)-(x-y) k)} m_{-}^{0}(x, k) m_{+}^{0}(y, k) d k .
\end{aligned}
$$


In the sequel $\dot{g}(k)=\partial_{k} g(k)$. Korotyaev $[\mathrm{K}$ proves:

Lemma 5.2. Consider $E(k)$ for $k \in[\pi n(j), \pi n(j+1)]$. Then $\dot{E}(k)=0$ for $k=$ $n(j) \pi, n(j+1) \pi$ and $\dot{E}(k)>0$ in $] \pi n(j), \pi n(j+1)[$. In $[\pi n(j), \pi n(j+1)]$ the equation $\ddot{E}(k)=0$ admits exactly one solution $k_{j}$. We have $\left.k_{j} \in\right] \pi n(j), \pi n(j+1)[$ and $\dddot{E}\left(k_{j}\right) \neq 0$. We have $\dot{E}(k)>0$ in $\left.] \pi n\left(n_{0}\right), \infty\right)$ and $\ddot{E}(k)>c_{0}>0$ in $\left[\pi n\left(n_{0}\right), \infty\right)$ for some $c_{0}>0$.

Notice that $E(-k)=E(k)$.

Theorem 5.3. Set $e^{i t h_{0}}(x, y)=\mathcal{U}(t, x, y)+\mathcal{V}(t, x, y)$ with

$$
\begin{aligned}
& \mathcal{U}(t, x, y)=\int_{|k| \leq \pi n\left(n_{0}\right)} e^{i(t E(k)-(x-y) k)} m_{-}^{0}(x, k) m_{+}^{0}(y, k) d k, \\
& \mathcal{V}(t, x, y)=\int_{|k| \geq \pi n\left(n_{0}\right)} e^{i(t E(k)-(x-y) k)} m_{-}^{0}(x, k) m_{+}^{0}(y, k) d k .
\end{aligned}
$$

Then $|\mathcal{U}(t, x, y)| \leq C\langle t\rangle^{-\frac{1}{3}}$ and $|\mathcal{V}(t, x, y)| \leq C|t|^{-\frac{1}{2}}$.

Assuming Lemma 5.4 below, the proof is in [F1]. In [C2] Theorem 5.3 is extended to the case of infinitely many energy bands. In the case of finitely many energy bands we have:

Lemma 5.4. For all $\alpha \in \mathbb{N} \cup\{0\}$ there are constants $C_{\alpha}>0$ such that for $(x, y) \in$ $\mathbb{R}^{2}$ and for $k \neq \pm n(j) \pi, j=1, \ldots, n_{0}$, we have

$$
\left|\partial_{k}^{\alpha}\left(m_{+}^{0}(x, k) m_{-}^{0}(y, k)-1\right)\right| \leq C_{\alpha}\langle k\rangle^{-1-\alpha} .
$$

Proof (sketch). Cases $\alpha=0,1$ are stated and proved in [C2 in the case of a generic periodic potential $P(x)$, i.e. all gaps nonempty. The case with finitely many bands is much easier. In the finite bands case, there is an $M>0$ such that the $\alpha=0$ case is valid for all complex $k$ with $|k| \geq M$. Here we are using the fact that $m_{+}^{0}(x, k) m_{-}^{0}(y, k)$ is holomorphic in $\left(\mathcal{K} \cup \mathcal{K}^{*}\right) \cup(\mathbb{R} \backslash \mathcal{T})$ (see [C2]) and where $z \in \mathcal{K}^{*}$ if and only if $\bar{z} \in \mathcal{K}$. The inequalities for $\alpha \geq 1$ hold by the Cauchy integral formula.

In the sequel we will set $w_{0}(k)=W\left[\phi_{+}^{0}(x, k), \phi_{-}^{0}(x, k)\right]$.

Lemma 5.5. For $k \neq \pm n(j) \pi, j=1, \ldots, n_{0}$, we have $\dot{E}(k)=-i w_{0}(k)$.

Proof. The formula $\dot{E}(k)=\frac{2 \sin (k)}{\varphi(1, k) N^{2}(k)}$ is formula (3.1) of [F2]. By direct computation $W\left[\widetilde{\phi}_{+}^{0}(k), \widetilde{\phi}_{-}^{0}(k)\right]=i \frac{2 \sin (k)}{\varphi(1, k)}$. So Lemma 5.5 follows by the normalization in the definition of $\phi_{ \pm}^{0}(x, k)$.

\section{Addition to $h_{0}$ of a small potential $q(x) \in C_{0}^{\infty}(\mathbb{R})$}

We consider the operator $h=h_{0}+q(x)$ with $q(x) \in C_{0}^{\infty}(\mathbb{R})$ small. We consider solutions $f_{ \pm}(x, k)=e^{ \pm i k x} m_{ \pm}(x, k)$ of $h u=E(k) u$ with

$$
\lim _{x \rightarrow+\infty} \frac{m_{+}(x, k)}{m_{+}^{0}(x, k)}=1=\lim _{x \rightarrow-\infty} \frac{m_{-}(x, k)}{m_{-}^{0}(x, k)} .
$$

Lemma 6.1. Assume that $q(x) \in C_{0}^{\infty}(\mathbb{R})$. Then for any $k \in \mathcal{K}$ the equation

$$
\begin{gathered}
m_{ \pm}(x, k)=m_{ \pm}^{0}(x, k)-\int_{x}^{ \pm \infty} e^{\mp i k(x-t)} A(x, t, k) q(t) m_{ \pm}(t, k) d t \\
\text { with } A(x, t, k):=\frac{\phi_{+}^{0}(x, k) \phi_{-}^{0}(t, k)-\phi_{-}^{0}(x, k) \phi_{+}^{0}(t, k)}{W\left[\phi_{+}^{0}(\cdot, k), \phi_{-}^{0}(\cdot, k)\right]},
\end{gathered}
$$


has a unique solution $m_{ \pm}(x, k)$ such that $f_{ \pm}(x, k)=e^{ \pm i k x} m_{ \pm}(x, k)$ solves hu= $E(k) u$ with the asymptotic property in (6.1). There is a constant $C_{q}$ such that for some $a>0$

$$
\begin{aligned}
& \left|m_{ \pm}(x, k)-m_{ \pm}^{0}(x, k)\right| \leq C_{q}\langle k\rangle^{-1} \max (1, \mp x) \\
& \left|\partial_{k}^{n} m_{ \pm}(x, k)\right| \leq C_{q}\langle k\rangle^{-1} \max ^{n+1}(1, \mp x) \text { for } n=1,2 \text { and } \Im k<a .
\end{aligned}
$$

Furthermore we have continuous extensions for $k \in[\pi n(j), \pi n(j+1)]$, for $k \in$ $\left[\pi n\left(n_{0}\right), \infty\right)$, for $k \in[-\pi n(j+1),-\pi n(j)]$ and for $k \in\left(-\infty,-\pi n\left(n_{0}\right)\right]$, where the above estimates continue to be satisfied.

Notice that estimates for $f_{ \pm}(x, k)$ are also in [F2]. Set

$$
D_{k}(x, t)=A(x, t, k) e^{-i k(x-t)}=\frac{m_{+}^{0}(x, k) m_{-}^{0}(t, k)-e^{-2 i k(x-t)} m_{-}^{0}(x, k) m_{+}^{0}(t, k)}{W\left[\phi_{+}^{0}(x, k), \phi_{-}^{0}(x, k)\right]} .
$$

Then Lemma 6.1 follows from standard arguments (see Lemma 1 of [DT]) by the following lemma:

Lemma 6.2. Let $t \geq x$. Then we have the following for a fixed $C>0$ :

(1) $\left|D_{k}(x, t)\right| \leq C\langle k\rangle^{-1}$ for $|k| \geq \pi n\left(n_{0}\right)+1$;

(2) $\left|D_{k}(x, t)\right| \leq C\langle x-t\rangle$;

(3) $\left|\dot{D}_{k}(x, t)\right| \leq C\langle x-t\rangle^{2}$ for $\dot{D}_{k}(x, t)=\partial_{k} D_{k}(x, t)$;

(4) $\left|\ddot{D}_{k}(x, t)\right| \leq C\langle x-t\rangle^{3}$ for $\ddot{D}_{k}(x, t)=\partial_{k}^{2} D_{k}(x, t)$.

(1) follows from Lemma 5.4, which yields a bound $\left|m_{ \pm}^{0}(x, k) m_{\mp}^{0}(t, k)\right| \leq C$ for a fixed $C$ and $w_{0}(k) \approx 2 i k$ for $|k| \nearrow \infty$. $w_{0}(k)=0$ iff $k=k_{0} \pm 0$ for $k_{0} \in \mathcal{T}$. By Lemmas 5.2 and $5.5 \dot{w}_{0}\left(k_{0} \pm 0\right) \neq 0$. Therefore, $\left|w_{0}(k)\right| \gtrsim \operatorname{dist}(k, \mathcal{T})$. For fixed $t \geq x$ consider $x_{1}, t_{1} \in[0,2]$ with $0 \leq t_{1}-x_{1} \leq t-x$ and $t-x=t_{1}-x_{1}+L$ with $L \in \mathbb{N}$. Then we can set $D_{k}(x, t)=D_{k}^{(1)}(x, t)+D_{k}^{(2)}(x, t)$ with

$$
\begin{aligned}
& D_{k}^{(1)}(x, t)=\frac{m_{+}^{0}\left(x_{1}, k\right) m_{-}^{0}\left(t_{1}, k\right)-e^{2 i k\left(t_{1}-x_{1}\right)} m_{-}^{0}\left(x_{1}, k\right) m_{+}^{0}\left(t_{1}, k\right)}{w_{0}(k)}, \\
& D_{k}^{(2)}(x, t)=e^{2 i k\left(t_{1}-x_{1}\right)} \frac{1-e^{2 i k L}}{w_{0}(k)} m_{-}^{0}\left(x_{1}, k\right) m_{+}^{0}\left(t_{1}, k\right) .
\end{aligned}
$$

$D_{k}^{(2)}(x, t)$ satisfies (1)-(4). Indeed Lemma 5.4 yields bounds on $m_{-}^{0}\left(x_{1}, k\right) m_{+}^{0}\left(t_{1}, k\right)$. Similarly, one can bound $\frac{1-e^{2 i k L}}{w_{0}(k)}$ using $\left|w_{0}(k)\right| \gtrsim \operatorname{dist}(k, \mathcal{T})$ and the fact that, for $k$ near $k_{0} \in \mathcal{T}, w_{0}(k)$ for $k \geq k_{0}$, resp. for $k \leq k_{0}$, admits an analytic extension defined around $k_{0}$; see [C2], [F1], [F2], [K]. Finally we claim that $\left|\partial_{k}^{n} D_{k}^{(1)}(x, t)\right| \leq C$ for $|n| \leq 2$ and a fixed $C$. For $k$ large this is a consequence of $w_{0}(k)=i \dot{E}(k) \approx 2 i k$ and the estimates on $E(k)$ (see $[\mathrm{K}]$ ) and Lemma 5.4. For $k_{0} \in \mathcal{T}$ and $k_{0}^{ \pm}=k_{0} \pm 0$

$$
e^{i k_{0} x} m_{+}^{0}\left(x, k_{0}^{+}\right) \equiv e^{-i k_{0} x} m_{-}^{0}\left(x, k_{0}^{+}\right) \text {and } e^{i k_{0} x} m_{+}^{0}\left(x, k_{0}^{-}\right) \equiv e^{-i k_{0} x} m_{-}^{0}\left(x, k_{0}^{-}\right) .
$$

Then both the numerator and denominator in the fraction defining $D_{k}^{(1)}(x, t)$ are 0 for $k=k_{0} \pm 0$ with $k_{0} \in \mathcal{T}$. Since, once again, these are functions admitting analytic extensions on fixed neighborhoods around $k_{0}$, one obtains the desired estimates $\left|\partial_{k}^{n} D_{k}^{(1)}(x, t)\right| \leq C$. 
Lemma 6.3. There exists $q(x) \in C_{0}^{\infty}(\mathbb{R})$ so that for all $k \in \mathcal{T}$ with $k>0$ we have

$$
\begin{aligned}
& \int_{\mathbb{R}} q(x)\left|m_{+}^{0}(x, 0)\right|^{2} d x>0 \\
& \mp \int_{\mathbb{R}} q(x)\left|m_{+}^{0}(x, k \pm 0)\right|^{2} d x>0 \text { for all } k \in \mathcal{T} \text { with } k>0 .
\end{aligned}
$$

Proof. $m_{+}^{0}(x, k \pm 0) \not \equiv 0$ for all $k \in \mathcal{T}$. Since $\sigma\left(h_{0}\right)$ has finitely many bands, the potential $P(x)$ is analytic, and hence also the $m_{ \pm}^{0}(x, k)$ are analytic in $x$. (2) can fail if and only if $\left|m_{+}^{0}\left(\cdot, k_{1}+0\right)\right|=\left|m_{+}^{0}\left(\cdot, k_{2}-0\right)\right|$ for two $k_{1}, k_{2} \geq 0$ in $\mathcal{T}$. The latter is the same as $\left|\phi_{+}^{0}\left(\cdot, k_{1}+0\right)\right|=\left|\phi_{+}^{0}\left(\cdot, k_{2}-0\right)\right|$. Notice that $E\left(k_{1}+0\right) \neq E\left(k_{2}-0\right)$, so one of them is nonzero. It is not restrictive to assume $E\left(k_{2}-0\right)>0$. Notice that $\phi_{+}^{0}\left(x, k_{1}+0\right)$ and $\phi_{+}^{0}\left(x, k_{2}-0\right)$ are real valued for $x \in \mathbb{R}$. Indeed, for instance, by definition $\widetilde{\widetilde{\phi}_{+}^{0}\left(x, k_{1}+0\right)}=\widetilde{\phi}_{-}^{0}\left(x, k_{1}+0\right)$ (see (2.5) of [C2]), $w_{0}\left(k_{1}+0\right)=0$ implies $\widetilde{\phi}_{+}^{0}\left(x, k_{1}+0\right)=C \widetilde{\phi}_{-}^{0}\left(x, k_{1}+0\right)$ for a fixed $C$ and by definition $\widetilde{\phi}_{+}^{0}\left(0, k_{1}+0\right)=$ $\widetilde{\phi}_{-}^{0}\left(0, k_{1}+0\right)=1$. By $E\left(k_{2}-0\right)>0$ the function $\phi_{+}^{0}\left(x, k_{2}-0\right)$ has infinitely many zeros (if it has 1 , it has infinitely many since $\phi_{+}^{0}\left(x, k_{2}-0\right)=e^{i k_{1} x} m_{+}^{0}\left(x, k_{2}-0\right)$ with $m_{+}^{0}\left(x, k_{2}-0\right)$ periodic in $x$, and if it has none, $\phi_{+}^{0}\left(x, k_{2}-0\right)$ would correspond to $\left.E\left(k_{2}\right)=0\right)$. We choose two points $a<b$ with $\phi_{+}^{0}\left(a, k_{2}-0\right)=\phi_{+}^{0}\left(b, k_{2}-0\right)=0$ and $\phi_{+}^{0}\left(x, k_{2}-0\right) \neq 0$ for $a<x<b$. $\left|\phi_{+}^{0}\left(\cdot, k_{1}+0\right)\right|=\left|\phi_{+}^{0}\left(\cdot, k_{2}-0\right)\right|$ implies the same statement for $\phi_{+}^{0}\left(x, k_{1}+0\right)$. But then they are both ground states for the Dirichlet problem in $[a, b]$ for the operator $h$. Then $\phi_{+}^{0}\left(x, k_{1}+0\right)=C \phi_{+}^{0}\left(x, k_{2}-0\right)$ for fixed $C$, but this is impossible by $E\left(k_{1}+0\right) \neq E\left(k_{2}-0\right)$.

We pick $q(x) \in C_{0}^{\infty}(\mathbb{R})$ small as in Lemma 6.3. By $[\mathrm{FK}$ we have that $\sigma(h)=$ $\sigma\left(h_{0}\right)$, and in particular $h$ has no eigenvalues. Furthermore there are no resonances; that is, there are not nonzero solutions of any of the equations $h u=A_{j}^{ \pm} u$ for $j>0$ with $u \in L^{\infty}$. Combining the asymptotics in Lemma 6.1 and the analysis of the thresholds in FK we conclude:

Lemma 6.4. For the Wronskian we have $W\left[f_{+}(k), f_{-}(k)\right] \neq 0$ for all $k$ and $W\left[f_{+}(k), f_{-}(k)\right] \approx 2 i k$ for $|k| \rightarrow \infty$.

There are equalities

$$
\begin{aligned}
& f_{\mp}(x, k)=\frac{R_{ \pm}(k)}{T(k)} f_{ \pm}(x, k)+\frac{1}{T(k)} f_{ \pm}(x,-k), \\
& \overline{f_{ \pm}(x, k)}=f_{ \pm}(x,-k), \overline{T(k)}=T(-k), \overline{R_{ \pm}(k)}=R_{ \pm}(-k), \\
& |T(k)|^{2}+\left|R_{ \pm}(k)\right|^{2}=1, \quad T(k) \overline{R_{ \pm}}(k)+R_{\mp}(k) \overline{T(k)}=0,
\end{aligned}
$$

with $T(k)$ and $R_{ \pm}(k)$ smooth functions for $k \in \mathbb{R} \backslash \mathcal{T}$ with smooth extensions in $[\pi n(j), \pi n(j+1)],\left[\pi n\left(n_{0}\right), \infty\right),[-\pi n(j+1),-\pi n(j)]$ and $\left(-\infty,-\pi n\left(n_{0}\right)\right]$ and with $T(k)=0$ for all $k \in \mathcal{T}$ and $T(0)=0$. For $q(x)$ small

$$
\left|[T(k)-1]^{(m)}\right|+\left|R_{ \pm}^{(m)}(k)\right| \leq \delta /\langle k\rangle \text { for }|m| \leq 2 .
$$

Next define

$$
\psi(x, k)=\left\{\begin{array}{c}
\frac{1}{\sqrt{2 \pi}} T(k) f_{+}(x, k) \quad \text { for } \quad k \geq 0, \\
\frac{1}{\sqrt{2 \pi}} T(-k) f_{-}(x,-k) \text { for } \quad k<0 .
\end{array}\right.
$$

Then by standard arguments we have the following version of Theorem 5.1 for $h$; see Lemma 4.7 of [C3]. 
Lemma 6.5. For $\mathcal{F}_{h} f(k):=\int_{\mathbb{R}} \psi(x, k) f(x) d x$ we have

$$
\begin{aligned}
& \int_{\mathbb{R}}|f(y)|^{2} d y=\int_{\mathbb{R}}\left|\mathcal{F}_{h} f(k)\right|^{2} d k, \\
& f(x)=\int_{\mathbb{R}} \overline{\psi(x, k)} \mathcal{F}_{h} f(k) d k, \\
& \mathcal{F}_{h}(h f)(k)=E(k) \mathcal{F}_{h} f(k), \\
& e^{i t h}(x, y)=\int_{\mathbb{R}} e^{i t E(k)} \overline{\psi(x, k)} \psi(y, k) d k .
\end{aligned}
$$

We have:

Lemma 6.6 (Dispersive estimates). Set $e^{i t h}(x, y)=\mathcal{U}(t, x, y)+\mathcal{V}(t, x, y)$ with

$$
\begin{aligned}
& \mathcal{U}(t, x, y)=\int_{|k| \leq \pi n\left(n_{0}\right)} e^{i t E(k)} \overline{\psi(x, k)} \psi(y, k) d k, \\
& \mathcal{V}(t, x, y)=\int_{|k| \geq \pi n\left(n_{0}\right)} e^{i t E(k)} \overline{\psi(x, k)} \psi(y, k) d k .
\end{aligned}
$$

Then $|\mathcal{U}(t, x, y)| \leq C\langle t\rangle^{-\frac{1}{3}}$ and $|\mathcal{V}(t, x, y)| \leq C|t|^{-\frac{1}{2}}$ for a fixed $C>0$.

For $\mathcal{U}(t, x, y)$ the argument is the same as Theorem 5.3. For $\mathcal{V}(t, x, y)$ the proof follows along the lines of Lemmas 2.4 and 2.5 in Wd2 using [C2].

7. WAVE OPERATORS AND PARTIAL DiAgONALIZATION FOR $H_{\omega}$

We write $H_{\omega}=\sigma_{3}(h+\omega)+B^{*}(\omega) A$ with $A(x)=\langle x\rangle^{-\tau}$ with $\tau>3 / 2$ and with $B^{*}(x, \omega)$ a $C^{2}$ function in $(x, \omega)$ with values in the space of $2 \times 2$ real valued matrices. For any $\omega$ in some compact set $K$ there is a constant $c(K)>0$ and $\alpha>0$ such that $\left|e^{\alpha|x|} B^{*}(x, \omega)\right| \leq c_{m}(K) \forall x \in \mathbb{R}$. We have:

Proposition 7.1. Assume that $H_{\omega}$ does not have resonances at $\pm \omega$ and $\sigma_{e}\left(H_{\omega}\right)$ does not contain eigenvalues. Then there are isomorphism inverses of each other, $W(\omega): L_{x}^{2} \rightarrow L_{c}^{2}\left(H_{\omega}\right)$ and $Z(\omega): L_{c}^{2}\left(H_{\omega}\right) \rightarrow L_{x}^{2}$, defined as follows: for $u \in L_{x}^{2}$, and $v$ such that $\sigma_{3} v \in L_{c}^{2}\left(H_{\omega}\right)$,

$$
\langle W u, v\rangle=\langle u, v\rangle+\lim _{\varepsilon \rightarrow 0^{+}} \frac{1}{2 \pi i} \int_{-\infty}^{+\infty}\left\langle A R_{\sigma_{3}(h+\omega)}(\lambda+i \varepsilon) u, B R_{H_{\omega}^{*}}(\lambda+i \varepsilon) v\right\rangle d \lambda
$$

for $u \in L_{c}^{2}\left(H_{\omega}\right), v \in L_{x}^{2}$,

$$
\langle Z u, v\rangle=\langle u, v\rangle+\lim _{\varepsilon \rightarrow 0^{+}} \frac{1}{2 \pi i} \int_{-\infty}^{+\infty}\left\langle A R_{H_{\omega}}(\lambda+i \varepsilon) u, B R_{\sigma_{3}(h+\omega)}(\lambda+i \varepsilon) v\right\rangle d \lambda .
$$

Then $P_{c}\left(H_{\omega}\right) H_{\omega}=W \sigma_{3}(h+\omega) Z .\|W(\omega)\|_{B\left(L_{x}^{2}, L_{c}^{2}\left(H_{\omega}\right)\right)}$ and $\|Z(\omega)\|_{B\left(L_{c}^{2}\left(H_{\omega}\right), L_{x}^{2}\right)}$ are uniformly locally bounded in $\omega$. 
We need to show that there is a fixed $c>0$ such that $\forall \epsilon \neq 0$

$$
\begin{aligned}
& \int\left\|\langle x\rangle^{-\tau} R_{\sigma_{3}(h+\omega)}(\lambda+i \varepsilon) u\right\|_{L_{x}^{2}}^{2} d \lambda \leq c\|u\|_{L_{x}^{2}}^{2} \text { for all } u \in L_{x}^{2}, \\
& \int\left\|B R_{\sigma_{3}(h+\omega)}(\lambda+i \varepsilon) u\right\|_{L_{x}^{2}}^{2} d \lambda \leq c\|u\|_{2}^{2} \text { for all } u \in L_{x}^{2}, \\
& \int\left\|B R_{H_{\omega}^{*}}(\lambda+i \varepsilon) u\right\|_{L_{x}^{2}}^{2} d \lambda \leq c\|u\|_{L_{x}^{2}}^{2} \text { for all } u \in L^{2}\left(H_{\omega}^{*}\right):=\sigma_{3} L^{2}\left(H_{\omega}\right), \\
& \int\left\|\langle x\rangle^{-\tau} R_{H_{\omega}}(\lambda+i \varepsilon) u\right\|_{L_{x}^{2}}^{2} d \lambda \leq c\|u\|_{L_{x}^{2}}^{2} \text { for all } u \in L_{c}^{2}\left(H_{\omega}\right) .
\end{aligned}
$$

Let us first prove (1), (2). They are consequences of (5) for $\tau>3 / 2$ :

$$
\int\left\|\langle x\rangle^{-\tau} R_{h}(\lambda+i \varepsilon) u\right\|_{L_{x}^{2}}^{2} d \lambda \leq c\|u\|_{L_{x}^{2}}^{2} \text { for all } u \in L_{x}^{2} .
$$

By (5.3) in Theorem 5.1 of $[\mathrm{K}]$, (5) will follow from

$$
\left\|\langle x\rangle^{-\tau} R_{h}(z)\langle x\rangle^{-\tau}\right\|_{B\left(L_{x}^{2}, L_{x}^{2}\right)}<C \text { for all } z \text { with } 0<|\Im z| .
$$

Observe that for $\Im k \geq 0, \pm(x-y) \geq 0$ and setting $w(k)=W\left[f_{+}(k), f_{-}(k)\right]$,

$$
\langle x\rangle^{-\tau} R_{h}(E(k), x, y)\langle y\rangle^{-\tau}=e^{i k|x-y|}\langle x\rangle^{-\tau} m_{ \pm}(x, k) m_{\mp}(y, k)\langle y\rangle^{-\tau} w^{-1}(k) .
$$

By Lemma $6.1,\left|m_{ \pm}(x, k) m_{\mp}(y, k)\right| \lesssim\langle x\rangle\langle y\rangle$. By Lemma $6.4,|w(k)| \gtrsim\langle k\rangle$. Hence

$$
\langle x\rangle^{-\tau}\left|R_{h}(z, x, y)\right|\langle y\rangle^{-\tau} \leq C\langle z\rangle^{-\frac{1}{2}}\langle x\rangle^{1-\tau}\langle y\rangle^{1-\tau}
$$

for all $z$ with $\Im z \geq 0$, resp. $\Im z \leq 0$ (for $\Im z=0$ there are two different continuations). Then for all $z$ with $\Im z \geq 0$, resp. $\Im z \leq 0$,

$$
\left\|\langle x\rangle^{-\tau} R_{h}(z, x, y)\langle y\rangle^{-\tau}\right\|_{L_{x, y}^{2}} \leq C\langle z\rangle^{-\frac{1}{2}} .
$$

(8) implies (6). We now consider (3) and (4), and for definiteness we focus on (4). We have for $A=\langle x\rangle^{-\tau}$,

$$
A R_{H_{\omega}}(z) u=\left(1+A R_{\sigma_{3}(h+\omega)}(z) B^{*}\right)^{-1} A R_{\sigma_{3}(h+\omega)}(z) u .
$$

(8) implies $\left\|A R_{\sigma_{3}(h+\omega)}(z) B^{*}\right\|_{B\left(L^{2}, L^{2}\right)} \lesssim\langle z\rangle^{-1 / 2}$. By Fredholm theory there is a bounded 0 measure set $X \subset \mathbb{R}$ such that in $\mathbb{R} \backslash X$ the following limits exist:

$$
\lim _{\epsilon \searrow 0} R_{H_{\omega}}(\lambda+i \varepsilon)=R_{H_{\omega}}^{ \pm}(\lambda) \text { in } C_{l o c}^{0}\left(\mathbb{R} \backslash X, B\left(L_{x}^{2, \tau}, L_{x}^{2,-\tau}\right)\right) .
$$

A point $\lambda$ belongs to $X$ exactly if $\operatorname{ker}\left(1+A R_{\sigma_{3}(h+\omega)}^{\alpha}(\lambda) B^{*}\right) \neq 0$ in $L^{2}$ for $\alpha=+$ or - . The points in $X \backslash \sigma_{e}\left(H_{\omega}\right)$ are eigenvalues of $H_{\omega}$. This follows from (9). Furthermore, the hypothesis that $\sigma_{e}\left(H_{\omega}\right)$ contains no eigenvalues of $H_{\omega}$ implies that $X \backslash \sigma_{e}\left(H_{\omega}\right)$ is exactly the set of eigenvalues of $H_{\omega}$. The exponential decay of $H_{\omega}-\sigma_{3}\left(h_{0}+\omega\right)$ implies, by standard arguments, that $H_{\omega}$ has finitely many eigenvalues. Therefore for $z$ close to an eigenvalue of $H_{\omega}$ and for $u \in L_{c}^{2}\left(H_{\omega}\right)$ we have that $\left\|A R_{H_{\omega}}(z) u\right\|_{L_{x}^{2}} \leq c\left\|R_{H_{\omega}}(z) u\right\|_{L_{x}^{2}} \leq c^{\prime}\|u\|_{L_{x}^{2}}$ for fixed constants. We will show below that $X \cap \sigma_{e}\left(H_{\omega}\right)$ is empty. Notice that this yields (4). To see this let $N \subset \mathbb{R}$ be the neighborhood of $\sigma_{e}\left(\mathcal{H}_{\omega}\right)$ formed by the points with distance $<\delta$ from $\sigma_{e}\left(\mathcal{H}_{\omega}\right)$, with $\delta>0$ a small number. We split the integral in (4) into two integrals with domain $\mathbb{R} \backslash N$ and $N$. The integral with domain $\mathbb{R} \backslash N$ is bounded, since for $\lambda \in \mathbb{R} \backslash N$ we have $\left\|\langle x\rangle^{-\tau} R_{H_{\omega}}(\lambda+i \varepsilon) u\right\|_{2} \leq\left\|R_{H_{\omega}}(\lambda+i \varepsilon) u\right\|_{2} \leq C\|u\|_{2}$ for 
a fixed $C$ and $\mathbb{R} \backslash N$ is a bounded set. To bound the integral with domain $N$ we use formula (9) above where $H_{0}=\sigma_{3}(h+\omega)$ :

$$
\langle x\rangle^{-\tau} R_{H_{\omega}}(\lambda+i \varepsilon) u=\left(1+\langle x\rangle^{-\tau} R_{H_{0}}(\lambda+i \varepsilon) B^{*}\right)^{-1}\langle x\rangle^{-\tau} R_{H_{0}}(\lambda+i \varepsilon) u .
$$

Then for any $u \in L^{2}$

$$
\begin{array}{r}
\left\|\langle x\rangle^{-\tau} R_{H_{\omega}}(\lambda+i \varepsilon) u\right\|_{L_{\lambda}^{2}\left(N, L_{x}^{2}\right)} \leq\left\|\left(1+\langle x\rangle^{-\tau} R_{H_{0}}(\lambda+i \varepsilon) B^{*}\right)^{-1}\right\|_{L_{\lambda}^{\infty}\left(N, B\left(L_{x}^{2}, L_{x}^{2}\right)\right)} \\
\left\|\langle x\rangle^{-\tau} R_{H_{0}}(\lambda+i \varepsilon) u\right\|_{L_{\lambda}^{2}\left(N, L_{x}^{2}\right)} .
\end{array}
$$

The first factor on the right-hand side of (10) is uniformly bounded by the hypothesis that $X \cap \sigma_{e}\left(H_{\omega}\right)$ is empty, while the second factor on the right-hand side of (10) is bounded for any $u \in \ell^{2}$ by (1). This yields (4), with the proof of (3) being similar.

Now we return to the set $X$. Our aim is to show that $X$ is formed exactly by the eigenvalues of $H_{\omega}$. It is enough to show that $X \cap \sigma_{e}\left(\mathcal{H}_{\omega}\right)$ is empty. We will proceed in two steps. We will first show that by (H7) $X$ does not contain any of these extremes thresholds of $\sigma_{3}(h+\omega)$, Lemma 7.2. We will then show that the points of $X$ in the interior of the spectral bands are necessarily eigenvalues of $H_{\omega}$. Since such "embedded" eigenvalues do not exist by (H7), we can conclude that $X \cap \sigma_{e}\left(\mathcal{H}_{\omega}\right)$ is empty.

Lemma 7.2. $X$ does not contain extremes of the spectral bands of $\sigma_{3}(h+\omega)$.

Proof. Suppose the claim is wrong and that $\lambda \in X$ is an extremum of one of the spectral bands of $\sigma_{3}(h+\omega)$, and pick $w \neq 0$ in $L^{2}$ such that

$$
\left(1+A R_{\sigma_{3}(h+\omega)}(\lambda) B^{*}\right) w=0 .
$$

Then $\left(1+B^{*} A R_{\sigma_{3}(h+\omega)}(\lambda)\right) B^{*} w=0$. Then $\psi=R_{\sigma_{3}(h+\omega)}(\lambda) B^{*} w$ is $\psi \neq 0$ and by standard arguments is a nontrivial distributional solution of $\left(H_{\omega}-\lambda\right) u=0$. We claim that

$$
\psi \in L_{x}^{\infty} .
$$

If (10) is true, we get a contradiction with hypothesis (H7). The proof of (10) reduces to show that for a rapidly decreasing bounded function $g(y)$ we have

$$
\|f\|_{L_{x}^{\infty}}<\infty \text { for } f(x)=\int_{\mathbb{R}} R_{h}(x, y, \lambda) g(y) d y .
$$

This is a consequence of Lemmas 6.1 and 6.4 and, for $w(k)=W\left[f_{+}(k), f_{-}(k)\right]$, of

$$
f(x)=f_{+}(x, k) \int_{-\infty}^{x} f_{-}(y, k) g(y) d y / w(k)+f_{-}(x, k) \int_{x}^{\infty} f_{-}(y, k) g(y) d y / w(k) .
$$

This proves Lemma 7.2.

Lemma 7.3. For any $\lambda_{0}$ in the interior of $\sigma_{e}\left(H_{\omega}\right)$ there are an open interval $I \subset \sigma_{e}\left(H_{\omega}\right)$ with $\lambda_{0} \in I$ and a constant $C$ such that $\|A R(\lambda \pm i \varepsilon) u\|_{L_{\lambda}^{2}(I) L_{x}^{2}} \leq C\|u\|_{L_{x}^{2}}$ for any $u$.

Proof. Let $A B_{1}^{*}=A B^{*}-\sigma_{3} q$. Then $H_{\omega}=\sigma_{3}\left(h_{0}+\omega\right)+A B_{1}^{*}$. The points $\lambda_{0} \in$ $\sigma\left(\sigma_{3}\left(h_{0}+\omega\right)\right)$, where $\|A R(\lambda \pm i \varepsilon) u\|_{L_{\lambda}^{2}(I) L_{x}^{2}}$ is unbounded for $I$ a small neighborhood of $\lambda_{0}$, are such that if we set

$$
A R_{H_{\omega}}^{ \pm}(\lambda) u=\left(1+A R_{\sigma_{3}\left(h_{0}+\omega\right)}^{ \pm}(\lambda) B_{1}^{*}\right)^{-1} A R_{\sigma_{3}\left(h_{0}+\omega\right)}(\lambda) u
$$


we have for one of the two signs

$$
\operatorname{ker}\left(1+A R_{\sigma_{3}\left(h_{0}+\omega\right)}^{ \pm}\left(\lambda_{0}\right) B_{1}^{*}\right) \neq 0 .
$$

By a standard argument (see $\S 2$ of [CPV]), if we assume Lemma 7.4 below we conclude that such a point $\lambda_{0}$ is an eigenvalue of $H_{\omega}$. Since we are excluding eigenvalues inside the spectral bands of $\sigma_{e}\left(H_{\omega}\right)$, we are done with the proof Lemma 7.3 .

Lemmas 7.2 and 7.3 imply that $X$ coincides with $\sigma_{d}\left(H_{\omega}\right) \cap \mathbb{R}$. Moreover the above arguments imply that the following limits exist:

$$
\lim _{\epsilon \searrow 0} R_{H_{\omega}}(\lambda \pm i \varepsilon)=R_{H_{\omega}}^{ \pm}(\lambda) \text { in } C^{0}\left(\sigma_{e}\left(H_{\omega}\right), B\left(L_{x}^{2, \tau}, L_{x}^{2,-\tau}\right)\right) .
$$

Lemma 7.4. Fix $E_{0}$ in the interior of a spectral band of $h_{0}$. Suppose that there is a function $\psi(x)$ such that $\psi \in L_{x}^{2, s}$ for all $s>0$ and $\mathcal{F}_{h_{0}}(\psi)\left( \pm k_{0}\right)=0$, where $E_{0}=E\left(k_{0}\right)$. Then $R_{h_{0}}^{ \pm}\left(E_{0}\right) \psi \in L_{x}^{2}$.

Proof. Consider a cutoff $\chi(E) \in C_{0}^{\infty}(\mathbb{R})$ with $\chi(E)=1$ for $E$ close to $E_{0}$ and $\chi(E)=0$ if $\left|E-E_{0}\right| \geq \varepsilon_{0}>0$ for $\varepsilon_{0}$ a small fixed number. Then

$$
R_{h_{0}}^{ \pm}\left(E_{0}\right) \psi=R_{h_{0}}^{ \pm}\left(E_{0}\right) \chi\left(h_{0}\right) \psi+R_{h_{0}}^{ \pm}\left(E_{0}\right)\left(1-\chi\left(h_{0}\right)\right) \psi .
$$

The second term on the right is in $L_{x}^{2}$. With the notation in $\S 5$ we have

$$
\mathcal{F}_{h_{0}}\left(\chi\left(h_{0}\right) \psi\right)(k)=\int_{\mathbb{R}} e^{i k x} m_{+}^{0}(x, k) \chi(E(k)) \psi(x) d x .
$$

Notice that $m_{+}^{0}(x, k) \chi(E(k))$ is the symbol of a smoothing pseudodifferential operator. This implies that $\partial_{k}^{n} \mathcal{F}_{h_{0}}\left(\chi\left(h_{0}\right) \psi\right) \in L_{k}^{2}$ for all $n$ by Lemma 5.4. Since $k_{0} \notin \mathcal{T}$, $\dot{E}\left(k_{0}\right) \neq 0$ by Lemma 5.2. Then also

$$
\partial_{k}^{n}\left[\frac{\mathcal{F}_{h_{0}}(\psi)(k) \chi(E(k))}{E(k)-E_{0}}\right] \in C^{0} \cap L_{k}^{1} \text { for all } n .
$$

We have

$$
R_{h_{0}}^{ \pm}\left(E_{0}\right) \chi\left(h_{0}\right) \psi(x)=\int_{\mathbb{R}} e^{-i k x} m_{-}^{0}(x, k) \frac{\mathcal{F}_{h_{0}}(\psi)(k)}{E(k)-E_{0}} \chi(E(k)) d k .
$$

Integrating by parts we conclude $x^{N} R_{h_{0}}^{ \pm}\left(E_{0}\right) \chi\left(h_{0}\right) \psi(x) \in L_{x}^{\infty}$ for all $N$.

By Proposition 7.1 and by the spectral theorem we conclude:

Lemma 7.5. For any $u \in L_{x}^{2}$ we have

$$
P_{c}\left(H_{\omega}\right) u=\lim _{M \rightarrow \infty} \lim _{\epsilon \rightarrow 0^{+}} \frac{1}{2 \pi i} \int_{\sigma_{e}\left(H_{\omega}\right)} \chi_{[-M, M]}(\lambda)\left[R_{H_{\omega}}(\lambda+i \epsilon)-R_{H_{\omega}}(\lambda-i \epsilon)\right] u d \lambda .
$$

Finally, we obtain the limiting absorption principle:

Lemma 7.6. For any $u \in L_{x}^{2, \tau}$ with $\tau>3 / 2$ we have

$$
P_{c}\left(H_{\omega}\right) u=\lim _{M \rightarrow \infty} \frac{1}{2 \pi i} \int_{\sigma_{e}\left(H_{\omega}\right)} \chi_{[-M, M]}(\lambda)\left[R_{H_{\omega}}^{+}(\lambda)-R_{H_{\omega}}^{-}(\lambda)\right] u d \lambda .
$$


Proof. For $u \in L_{x}^{2, \tau}$ and for fixed $M$ the $\epsilon \rightarrow 0^{+}$limit in Lemma 7.5 converges in $L_{x}^{2,-\tau}$ to the integral in Lemma 7.6.

\section{Plane waves for $H_{\omega}$}

We recall $H_{\omega}=\sigma_{3}\left(h_{0}+\omega\right)+V(x)$ with:

- $V(x)$ a real entries square 2 matrix s.t. $\sigma_{1} V(x)=-V(x) \sigma_{1}$ and $\sigma_{3} V(x)=$ ${ }^{t} V(x) \sigma_{3}$;

- $|V(x)| \leq C e^{-\sqrt{2 \omega}|x|}$;

- $H_{\omega}$ has no eigenvalues in $\sigma_{e}\left(H_{\omega}\right)$, and the points and the thresholds of the spectral bands of $\sigma_{e}\left(H_{\omega}\right)$ are not resonances. This last statement means that for any $k \in \mathcal{T}$ and for $\lambda=E(k \pm 0)+\omega)$, as well as for $\lambda=-E(k \pm 0)$ $-\omega$, if $g \in L_{x}^{\infty}$ satisfies $H_{\omega} g=\lambda g$, then $g=0$.

We set

$$
\eta(x)=\int_{x}^{\infty}|V(t)| d t, \quad \gamma(x)=\int_{x}^{\infty}\langle t\rangle|V(t)| d t .
$$

We have $\sigma_{e}\left(H_{\omega}\right)=\left(\omega+\sigma\left(h_{0}\right)\right) \cup\left(-\omega-\sigma\left(h_{0}\right)\right)$. Because of the symmetries of $H_{\omega}$ we look at Jost functions $F_{ \pm}(x, k)$ close to $\omega+\sigma\left(h_{0}\right)$.

For $\lambda \notin \sigma_{e}\left(H_{\omega}\right)$ let $k \in \mathbb{C}$ with $\Im k \geq 0$ such that $\lambda=E(k)+\omega$ (in the sequel, for $\lambda$ and $k$ in the same sentence, we will always have the relation $\lambda=E(k)+\omega)$. We now consider solutions of $H_{\omega} u=\lambda u$ of the following form, where $A(x, t, k)$ is given by (6.4):

$$
\begin{aligned}
F_{ \pm}(x, k) & =\phi_{ \pm}^{0}(x, k) \vec{e}_{1}-\int_{x}^{ \pm \infty} A(x, t, k) \operatorname{diag}(1,0) V(t) F_{ \pm}(t, k) d t \\
& -\int_{\mathbb{R}} R_{h_{0}}(-2 \omega-E(k), x, t) \operatorname{diag}(0,1) V(t) F_{ \pm}(t, k) d t .
\end{aligned}
$$

Lemma 8.1. Assume (H7) and (H9). Then there is a small $\delta>0$ and a finite set $S \subset \overline{\mathbb{C}}_{+}$such that for $k \notin S$ with $0 \leq \Im k \leq \delta$, (8.1) has for any choice of sign a unique solution satisfying the estimates listed below for $M_{ \pm}(x, k):=e^{\mp i k x} F_{ \pm}(x, k)$. These solutions solve $H_{\omega} u=\lambda u$ with $\lambda=\omega+E(k)$ and with the asymptotic property $F_{ \pm}(x, k)=\phi_{ \pm}^{0}(x, k) \vec{e}_{1}+o\left(e^{ \pm i k x}\right)$ for $x \rightarrow \pm \infty$. For any fixed $0<a<\sqrt{2 \omega}$ and any $\varepsilon>0$ there is $C_{\varepsilon}$ such that $\forall x \in \mathbb{R}$ and $\forall k \in \mathbb{C}$ with $0 \leq \Im k \leq \delta$ and $\operatorname{dist}(k, S)>\varepsilon>0$ we have:

$$
\begin{aligned}
& \left|M_{ \pm}(x, k)-m_{ \pm}^{0}(x, k) \vec{e}_{1}\right| \leq C_{\varepsilon} e^{-a \max (1, \mp x)}\langle k\rangle^{-1}(1+\max (1, \mp x)) ; \\
& \left|\partial_{k}\left[M_{ \pm}(x, k)-m_{ \pm}^{0}(x, k) \vec{e}_{1}\right]\right| \leq C_{\varepsilon} \max ^{2}(1, \mp x) ; \\
& \left|\partial_{k}^{2}\left[M_{ \pm}(x, k)-m_{ \pm}^{0}(x, k) \vec{e}_{1}\right]\right| \leq C_{\varepsilon} \max ^{3}(1, \mp x) .
\end{aligned}
$$

Proof. We sketch the proof for the + case and drop the index. Using the notation in $\S 6$,

$$
\begin{aligned}
M(x, k)= & m_{+}^{0}(x, k) \vec{e}_{1}-\int_{x}^{\infty} D_{k}(x, t) \operatorname{diag}(1,0) V(t) M(t, k) d t \\
& -\int_{\mathbb{R}} R_{h_{0}}(-2 \omega-E(k), x, t) e^{-i k(x-t)} \operatorname{diag}(0,1) V(t) M(t, k) d t .
\end{aligned}
$$


We have $\left|R_{h_{0}}(-2 \omega-E(k), x, t) e^{-i k(x-t)}\right| \leq C e^{-\sqrt{2 \omega}|x-t|}\langle k\rangle^{-1}$. For $V=\left\{V_{\ell, j}\right\}$ let

$$
\begin{aligned}
& { }^{t} M=\left(M_{1}, M_{2}\right), \quad M_{1}^{(1)}(x, k):=-\int_{x}^{\infty} D_{k}(x, t) V_{12}(t) M_{2}(t, k) d t, \\
& M_{1}^{(0)}(x, k):=m_{+}^{0}(x, k)-\int_{x}^{\infty} D_{k}(x, t) V_{11}(t) M_{1}(t, k) d t .
\end{aligned}
$$

Then $M_{1}(x, k)=M_{1}^{(0)}(x, k)+M_{1}^{(1)}(x, k)$. By standard arguments:

Lemma 8.2. For given $M_{2} \in L_{x}^{\infty}$ for some fixed $C=C(V)$ we have

$$
\begin{aligned}
& \left|M_{1}^{(0)}(x, k)-m_{+}^{0}(x, k)\right| \leq C \max (1,-x) \gamma(x)\langle k\rangle^{-1} ; \\
& \left|M_{1}^{(1)}(x, k)\right| \leq C \max (1,-x) \gamma(x)\langle k\rangle^{-1}\left\|M_{2}(\cdot, k)\right\|_{L_{x}^{\infty} .} .
\end{aligned}
$$

Continuation of proof of Lemma 8.1. We have $M_{1}^{(1)}(x, k)=L(k) M_{2}(x, k)$ with $L(k)$ a linear operator such that for a fixed $C$

$$
\left|L(k) M_{2}(x)\right| \leq C\langle k\rangle^{-1} \max (1,-x)\left\|M_{2}(\cdot, k)\right\|_{L_{x}^{\infty}} .
$$

Eliminate $M_{1}$ from (8.2) to get the following system for $K(k)$ defined below:

$$
\begin{gathered}
(1+K(k)) M_{2}(x, k)=-\int_{\mathbb{R}} R_{h_{0}}(-2 \omega-E(k), x, t) e^{-i k(x-t)} V_{21}(t) M_{1}^{(0)}(t, k) d t \\
K(k) g(x)=\int_{\mathbb{R}} R_{h_{0}}(-2 \omega-E(k), x, t) e^{-i k(x-t)}\left(V_{21}(t) L(k)[g](t)+V_{22}(t) g(t)\right) d t .
\end{gathered}
$$

Lemma 8.3. Let $0 \leq \Im k<\delta<\sqrt{2 \omega}-a$ for some $a>0$. Then $K(k)$ maps $L_{x}^{\infty}$ in $e^{-a|x|} W_{x}^{1, \infty}$. There is a finite set $S \subset \overline{\mathbb{C}}_{+}$such that for $k$ as above with $k \notin S$, equation (8.3) admits a unique solution $M_{2}(x, k) \in L_{x}^{\infty}$. Furthermore, for any $\varepsilon>0$ there is a $C_{\varepsilon}$ such that if $\operatorname{dist}(k, S)>\varepsilon$, then

$$
\left|e^{a|x|} M_{2}(x, k)\right| \leq C_{\varepsilon}\langle k\rangle^{-1} \text {. }
$$

Proof. The fact that $K(k): L_{x}^{\infty} \rightarrow e^{-a|x|} W_{x}^{1, \infty}$ if $0 \leq \Im k<\delta<\sqrt{2 \omega}-a$ is an elementary computation. For $|k| \rightarrow \infty$ we have $\|K(k)\|_{B\left(L_{x}^{\infty}, L_{x}^{\infty}\right)} \rightarrow 0$, so we can solve (8.3) by obtaining (1). $K(k): L_{x}^{\infty} \rightarrow L_{x}^{\infty}$ is a compact operator. For $|k| \lesssim 1$ by the Fredholm alternative, $S$ will be formed by the $k$ with $\operatorname{ker}(1+K(k)) \neq 0$. The set $S$ is necessarily discrete and contained in $|k| \lesssim 1$. We start by considering $k \in S \cap \mathbb{R}$. If we have such a $k$, then we have a nonzero solution of the equation obtained from (8.3) by replacing $M_{1}^{(0)}(t, k)$ with 0 . Going backwards in the above argument, we obtain the existence of a nontrivial $g \in L_{x}^{\infty}$ satisfying

$$
\begin{aligned}
g(x) & =-\int_{x}^{\infty} A(x, t, k) \operatorname{diag}(1,0) V(t) g(t) d t \\
& -\int_{\mathbb{R}} R_{h_{0}}(-2 \omega-E(k), x, t) \operatorname{diag}(0,1) V(t) g(t) d t .
\end{aligned}
$$

Such $g \in L_{x}^{\infty}$ is a distributional solution of $H_{\omega} g=\lambda g$ with $\lambda=E(k)+\omega$. By hypothesis we are assuming such solutions do not exist for $\lambda=E(k \pm 0)+\omega$ with $k \in \mathcal{T}$, that is, for $\lambda$ a threshold in $\sigma_{e}\left(H_{\omega}\right)$. By continuity, $\operatorname{ker}(1+K(k))=0$ near such thresholds. Since the points in $\mathcal{T}$ are the only possible accumulation points of $S$, we conclude that $S$ is finite. 
Continuation of proof of Lemma 8.1. Lemmas 8.2-8.3 imply (1) in Lemma 8.1. To prove (2) we differentiate (8.2) in $k$ obtaining for the dot representing $\partial_{k}$, and for $E_{k}(x, t)=R_{h_{0}}(-2 \omega-E(k), x, t)$,

$$
\begin{aligned}
& \dot{M}(x, k)=\vec{e}_{1} \dot{m}_{+}^{0}(x, k) \\
& -\left(\int_{x}^{\infty} D_{k}(x, t) \operatorname{diag}(1,0)+\int_{\mathbb{R}} E_{k}(x, t) \operatorname{diag}(0,1)\right) V(t) \dot{M}(t, k) d t \\
& -\left(\int_{x}^{\infty} \dot{D}_{k}(x, t) \operatorname{diag}(1,0)+\int_{\mathbb{R}} \dot{E}_{k}(x, t) \operatorname{diag}(0,1)\right) V(t) M(t, k) d t .
\end{aligned}
$$

We have $\left|\partial_{k}^{j} E_{k}(x, t)\right| \leq C e^{-\alpha\langle k\rangle|x-t|}$ for $j=0,1$ for fixed $C>0$ and $\alpha>0$. We write $\dot{M}_{1}(x, k)=\dot{M}_{1}^{(0)}(x, k)+\dot{M}_{1}^{(1)}(x, k)$, with $\dot{M}_{1}^{(1)}(x, k)=L(k) \dot{M}_{2}(x, k)$ and with

$$
\begin{aligned}
& \dot{M}_{1}^{(0)}(x, k) \vec{e}_{1}=\vec{e}_{1} \dot{m}_{+}^{0}(x, k) \\
& +(\text { third line of }(4))-\vec{e}_{1} \int_{x}^{\infty} D_{k}(x, t) V_{12}(t) \dot{M}_{2}(t, k) d t .
\end{aligned}
$$

Then

$$
\begin{aligned}
& \left|\dot{M}_{1}^{(0)}(x, k)\right| \leq C \max ^{2}(1,-x) \text { and } \\
& \left|L(k) \dot{M}_{2}(x, k)\right| \leq C\langle k\rangle^{-1} \max (1,-x)\left\|\dot{M}_{2}(\cdot, k)\right\|_{L_{x}^{\infty}} .
\end{aligned}
$$

We obtain an analogue of system $(8.3)$, with $M_{1}^{(0)}(x, k)$ replaced by $\dot{M}_{1}^{(0)}(x, k)$. Then we conclude $\left|e^{a|x|} \dot{M}_{2}(x, k)\right| \leq \frac{C}{1+|k|}$. Repeating the argument in a similar way, we get claim (3) in Lemma 8.1.

If $S \cap \mathbb{R}=\emptyset$, by taking $\delta$ small enough we can neglect the set $S$. However, in Lemma 5.4 of [C1] the proof that $S \cap \mathbb{R}=\emptyset$ is wrong. In fact, the discussion in KS] allows for the existence of $S$. So let us now assume $S \cap \mathbb{R} \neq \emptyset$. For $k$ near $S \cap \mathbb{R}$ we consider the system

$$
\left(1+R_{\sigma_{3}\left(h_{0}+\omega\right)}^{+}(\lambda) V\right) \Phi_{ \pm}(\cdot, k)=\phi_{ \pm}^{0}(x, k) \vec{e}_{1} .
$$

Lemma 8.4 . Let $\lambda=E(k)+\omega$ with $E(k) \in \sigma\left(h_{0}\right)$, with $k \neq \pm \pi n(j)$ for all $j$.

(1) For any choice of signs, system (8.5) admits exactly one solution in $L_{x}^{\infty}$.

(2) For $k \notin S$ we have $\Phi_{ \pm}(x, k)=c_{ \pm}(k) F_{ \pm}(x, k)$ for some constants $c_{ \pm}(k)$.

(3) Fix a decomposition $V=B^{*} A$ with $A$ and $B^{*} \in C^{2}$ and exponentially decreasing. Then $k \rightarrow A \Phi_{ \pm}(x, k) \in L_{x}^{2}$ is a real analytic map for $k$ near $S$.

(4) $c_{ \pm}(k)$ are real analytic functions in $k$ for $k$ near $S$.

Proof. For definiteness pick + . For $\lambda=E+\omega$, by standard arguments

$$
R_{\sigma_{3}\left(h_{0}+\omega\right)}^{+}(\lambda) V \text { is a } L_{x}^{\infty} \rightarrow L_{x}^{\infty} \text { compact operator. }
$$

By (5) and the Fredholm theory we know that (8.5) has a unique solution unless there is a nonzero solution $g \in L_{x}^{\infty}$ of

$$
\left(1+R_{\sigma_{3}\left(h_{0}+\omega\right)}^{+}(\lambda) V\right) g=0 .
$$

Notice that ${ }^{t} g=\left(g_{1}, g_{2}\right)$,

$$
H_{\omega} g=\lambda g \text { in the sense of distributions, }
$$

and that $\left|g_{2}(x)\right| \leq C e^{-\sqrt{2 \omega}|x|}$ for some $C>0$. By (6) we obtain

$$
\left\langle\left(1+R_{\sigma_{3}\left(h_{0}+\omega\right)}^{+}(\lambda) V\right) g, \sigma_{3} V g\right\rangle=0 .
$$


Then

$$
\left\langle\delta\left(h_{0}-E(k)\right)\left(V_{11} g_{1}+V_{12} g_{2}\right), V_{11} g_{1}+V_{12} g_{2}\right\rangle=0 .
$$

Notice that $V_{11} g_{1}+V_{12} g_{2} \in L_{x}^{2, s}$ for any $s \in \mathbb{R}$. For $\psi \in L_{x}^{2, s}$ with $s>1 / 2$ we have

$$
\left\langle\delta\left(h_{0}-E(k)\right) \psi, \psi\right\rangle=|\dot{E}(k)|^{-1}\left(\left|\mathcal{F}_{h_{0}} \psi(k)\right|^{2}+\left|\mathcal{F}_{h_{0}} \psi(-k)\right|^{2}\right) .
$$

So if we set $\psi=V_{11} g_{1}+V_{12} g_{2}$, then $\mathcal{F}_{h_{0}} \psi( \pm k)=0$. We can apply Lemma 7.4 and conclude that

$$
g_{1}=R_{h_{0}}^{+}(E(k)) \psi \in L^{2}(\mathbb{R}) .
$$

So $g \in L_{x}^{2}$ and (7) imply that $g$ is an eigenfunction and that $\lambda$ is an eigenvalue. Since we are excluding this possibility, we conclude that there are non nonzero solutions of (6) and that claim (1) is correct.

From (8.5) we get for $w_{0}(k)=W\left[\phi_{+}^{0}(k), \phi_{-}^{0}(k)\right]$,

$$
\begin{aligned}
& \Phi_{ \pm}(x, k)=\phi_{ \pm}^{0}(x, k) \vec{e}_{1}, \\
& +\phi_{+}^{0}(x, k) \int_{-\infty}^{x} \phi_{-}^{0}(y, k) \operatorname{diag}(1,0) V(y) \Phi_{ \pm}(y, k) d y / w_{0}(k) \\
& -\phi_{-}^{0}(x, k) \int_{x}^{\infty} \phi_{+}^{0}(y, k) \operatorname{diag}(1,0) V(y) \Phi_{ \pm}(y, k) d y / w_{0}(k) \\
& -\int_{\mathbb{R}} R_{h_{0}}(\omega-E(k), x, y) \operatorname{diag}(0,1) V(y) \Phi_{ \pm}(y, k) d y .
\end{aligned}
$$

Then, for

$$
\begin{aligned}
& c_{+}(k)=1+\int_{\mathbb{R}} \phi_{-}^{0}(y, k)\left(V_{11}(y) \Phi_{1+}(y, k)+V_{12}(y) \Phi_{2+}(y, k)\right) d y / w_{0}(k), \\
& c_{-}(k)=1-\int_{\mathbb{R}} \phi_{+}^{0}(y, k)\left(V_{11}(y) \Phi_{1-}(y, k)+V_{12}(y) \Phi_{2-}(y, k)\right) d y / w_{0}(k)
\end{aligned}
$$

and for $k \notin S$ we have for $g_{ \pm}(x):=\Phi_{ \pm}(x, k)-c_{ \pm}(k) F_{ \pm}(x, k)$,

$$
g \in L_{x}^{\infty} \text { with } \lim _{x \rightarrow \pm \infty} g_{ \pm}(x)=0 \text { and } H_{\omega} g_{ \pm}=\lambda g_{ \pm} .
$$

Since $k \notin S$ this yields $g_{ \pm} \equiv 0$.

To prove claims (3) and (4) we write (8.5) as

$$
\left(1+A R_{\sigma_{3}\left(h_{0}+\omega\right)}^{+}(\lambda) B^{*}\right) A \Phi_{ \pm}(\cdot, k)=A \phi_{ \pm}^{0}(x, k) \vec{e}_{1} .
$$

Since $A R_{\sigma_{3}\left(h_{0}+\omega\right)}^{+}(\lambda) B^{*} \in B\left(L_{x}^{2}, L_{x}^{2}\right)$ is real analytic in $k$, and similarly $k \rightarrow$ $A \phi_{ \pm}^{0}(x, k) \in L_{x}^{2}$ is real analytic in $k$, we obtain claims (3) and (4) by the chain rule.

Lemma 8.1 yields Jost functions $F(x, k)$ for $H_{\omega}$ for energy $\lambda$ close to $\omega+\sigma(h)$ and for $k \notin S$. By $\sigma_{1} H_{\omega}=-H_{\omega} \sigma_{1}$ we conclude that $\sigma_{1} F(x, k)$ are Jost functions for $H_{\omega}$ for energy $\lambda$ close to $-\omega-\sigma(h)$. From $\sigma_{3} H_{\omega}=H_{\omega}^{*} \sigma_{3}$ we get that $\sigma_{3} F(x, k)$ are Jost functions for $H_{\omega}^{*}$ for energy $\lambda$ close to $\omega+\sigma(h)$ and that $\sigma_{3} \sigma_{1} F(x, k)$ are Jost functions for $H_{\omega}^{*}$ for energy $\lambda$ close to $-\omega-\sigma(h)$. By elementary arguments we get:

Lemma 8.5. For $k \in \mathbb{R} \backslash S$ we have $\overline{F_{ \pm}(x, k)}=F_{ \pm}(x,-k)$, and for $k \neq 0$ we have

$$
F_{\mp}(x, k)=\frac{1}{T(k)} \overline{F_{ \pm}(x, k)}+\frac{R_{ \pm}(k)}{T(k)} F_{ \pm}(x, k),
$$

where $T(k)$ and $R_{ \pm}(k)$ are defined by the above formula. 
Lemma 8.6. All the Wronskians below are constant. We have for any $k \in \mathbb{R} \backslash S$,

$$
\begin{aligned}
& W\left[\overline{F_{ \pm}(k)}, F_{ \pm}(k)\right]=W\left[\phi_{\mp}^{0}(\cdot, k), \phi_{ \pm}^{0}(\cdot, k)\right], \\
& T(k)=\frac{W\left[\phi_{\mp}^{0}(\cdot, k), \phi_{ \pm}^{0}(\cdot, k)\right]}{W\left[F_{\mp}(k), F_{ \pm}(k)\right]}, \quad R_{ \pm}(k)=\frac{W\left[F_{\mp}(k), \overline{F_{ \pm}}(k)\right]}{W\left[F_{ \pm}(k), F_{\mp}(k)\right]}, \\
& \overline{T(k)}=T(-k), \overline{R_{ \pm}(k)}=R_{ \pm}(-k), \\
& |T(k)|^{2}+\left|R_{ \pm}(k)\right|^{2}=1, \quad T(k) \overline{R_{ \pm}(k)}+R_{\mp}(k) \overline{T(k)}=0 .
\end{aligned}
$$

Proof. For the fact that the Wronskians are constant see Lemma 5.8 KS. The rest of the proof is the same as Lemma 5.6 of [C1].

Lemma 8.7. For $k \in \mathbb{R}$ we have

$$
W\left[\Phi_{+}(k), \Phi_{-}(k)\right]=c_{+}(k) W\left[\phi_{+}^{0}(\cdot, k), \phi_{-}^{0}(\cdot, k)\right]=c_{-}(k) W\left[\phi_{+}^{0}(\cdot, k), \phi_{-}^{0}(\cdot, k)\right] .
$$

In particular, $c_{+}(k)=c_{-}(k)=T(k)$.

Proof. We have the asymptotic behaviors, with $\partial_{x} o(1)=o(1)$,

$$
\begin{aligned}
& \Phi_{+}(x, k)=c_{+}(k) \phi_{+}^{0}(x, k) \vec{e}_{1}+o(1) \text { for } x \rightarrow+\infty, \\
& \Phi_{-}(x, k)=\phi_{-}^{0}(x, k) \vec{e}_{1}+C_{1}(k) \phi_{+}^{0}(x, k) \vec{e}_{1}+o(1) \text { for } x \rightarrow+\infty, \\
& \Phi_{-}(x, k)=c_{-}(k) \phi_{-}^{0}(x, k) \vec{e}_{1}+o(1) \text { for } x \rightarrow-\infty, \\
& \Phi_{+}(x, k)=\phi_{+}^{0}(x, k) \vec{e}_{1}+C_{2}(k) \phi_{-}^{0}(x, k) \vec{e}_{1}+o(1) \text { for } x \rightarrow-\infty,
\end{aligned}
$$

with $c_{ \pm}(k)$ defined in Lemma 8.4 and for some constants $C_{j}(k)$ with $j=1,2$. In particular, for later use, $C_{1}(k)$ is smooth for $k$ near $S$ and given by

$$
C_{1}(k)=\int_{\mathbb{R}} \phi_{-}^{0}(y, k)\left(V_{11}(y) \Phi_{1-}(y, k)+V_{12}(y) \Phi_{2-}(y, k)\right) d y / w_{0}(k) .
$$

(1) follows by using these asymptotic expansions and the fact that the Wronskian $W\left[\Phi_{+}(k), \Phi_{-}(k)\right]$ is a constant. We have

$$
T(k)=\frac{W\left[\phi_{-}^{0}, \phi_{+}^{0}\right]}{W\left[F_{-}, F_{+}\right]}=c_{+} c_{-} \frac{W\left[\phi_{-}^{0}, \phi_{+}^{0}\right]}{W\left[\Phi_{-}, \Phi_{+}\right]}=\frac{c_{+} c_{-}}{c_{ \pm}}=c_{ \pm} .
$$

Lemma 8.8. $T(k)$ and $R_{ \pm}(k)$ are in $C^{1}(\mathbb{R})$, and there is $C>0$ such that for $n \leq 1$,

$$
\left|d^{n}[T(k)-1] / d k^{n}\right|+\left|d^{n} R_{ \pm}(k) / d k^{n}\right| \leq C /\langle k\rangle .
$$

Proof. Notice that for $k \notin T$ we have $W\left[\phi_{-}^{0}(k), \phi_{+}^{0}(k)\right] \neq 0$. So (4) of Lemma 8.6 and the nonresonance hypothesis at the thresholds imply $W\left[F_{-}, F_{+}\right] \neq 0$ in $\mathbb{R} \backslash S$. There $T(k)$ and $R_{ \pm}(k)$ are $C^{1}$ with the desired asymptotic estimates by Lemma 8.1. Near $S$ we use $T(k)=c_{ \pm}(k)$ and (3) of Lemma 8.4 to conclude that $T \in C^{1}(\mathbb{R})$. Near $S$

$$
R_{+}(k)=\frac{W\left[F_{-}, \bar{F}_{+}\right]}{W\left[F_{+}, F_{-}\right]}=\frac{\left|c_{+}\right|^{2}}{c_{+}^{2}} \frac{W\left[\Phi_{-}, \bar{\Phi}_{+}\right]}{W\left[\Phi_{+}, \Phi_{-}\right]}=C_{1}(k) \frac{\bar{c}_{+}^{2}}{c_{+}^{2}} .
$$

Here $C_{1}(k)$ is in (2) of Lemma 8.7 and is smooth. If $k_{0} \in S$, then there is some $m \geq$ such that $c_{+}^{(m)}\left(k_{0}\right) \neq 0$; then also $\bar{c}_{+} / c_{+}$is smooth near $k_{0}$. So $R_{+} \in C^{1}(\mathbb{R})$. The argument for $R_{-}$is similar. 
We consider the following system:

$$
\begin{aligned}
G_{ \pm}(x, k) & =\phi_{ \pm}^{0}\left(x, k_{1}\right) \vec{e}_{2}-\int_{x}^{ \pm \infty} A(x, t, k) \operatorname{diag}(1,0) V(t) G_{ \pm}(t, k) d t \\
& -\int_{x}^{ \pm \infty} A\left(x, t, k_{1}\right) \operatorname{diag}(0,1) V(t) g_{ \pm}(t, k) d t
\end{aligned}
$$

where $E\left(k_{1}\right)=-E(k)-2 \omega$. The proof of Lemmas 8.9 and 8.10 below follow by routine arguments.

Lemma 8.9. For $\lambda=\omega+E(k)$ with $0 \leq \Im k<\delta$, system (8.7) admits exactly one solution $G_{ \pm}(x, k)$ which satisfies $H_{\omega} G_{ \pm}=\lambda G_{ \pm} ; G_{ \pm}(x, k)$ is real for $k \in \mathbb{R}$. There is $C$ such that

$$
\begin{aligned}
& \left|G_{ \pm}(x, k)-\phi_{ \pm}^{0}\left(x, k_{1}\right) \vec{e}_{2}\right| \leq C\langle k\rangle^{-1}\left|\int_{x}^{ \pm \infty}\langle t\rangle\right| V(t)|d t| e^{\mp x \Re \sqrt{k^{2}+2 \omega}}, \\
& \left|\partial_{x} G_{ \pm}-\partial_{x} \phi_{ \pm}^{0}\left(x, k_{1}\right) \vec{e}_{2}\right| \leq C\left|\int_{x}^{ \pm \infty}\langle t\rangle\right| V(t)|d t| e^{\mp x \Re \sqrt{k^{2}+2 \omega}} .
\end{aligned}
$$

We have that $w(k)=\left[G_{-}(k), G_{+}(k)\right]$ is a continuous function with $w(k)=0$ if and only if $k \in S$. We have $w(k)=2 \sqrt{k^{2}+2 \omega}(1+o(1))$ for $|k| \rightarrow \infty$.

We have $\left[F_{ \pm}(x, k), G_{ \pm}(x, k)\right]=0$ for any $k \notin S$. For $\Im k>0$ and $g \in$ $L^{\infty}(b,+\infty)$, for some $b \in \mathbb{R}$ and with $H_{\omega} g=\lambda g, g(x)=\mu F_{+}(x, k)+\nu G_{+}(x, k)$ for constants $\mu$ and $\nu$. If $g \in L^{\infty}(-\infty, b)$ solves $H_{\omega} g=\lambda g$, then $g(x)=\mu F_{-}(x, k)+$ $\nu G_{-}(x, k)$.

Lemma 8.10. Let $\lambda=\omega+E(k)$ with $0<\Im k<\delta$ and $k \notin S$. The resolvent $R_{H_{\omega}}(\lambda)$ has an integral kernel given by $R_{H_{\omega}}(x, y, \lambda)=R_{1}(x, y)+R_{2}(x, y)$ where for $x<y$

$$
R_{1}(x, y)=-\frac{F_{-}(x, k)^{t}\left(\sigma_{3} F_{+}(y, k)\right)}{W\left[F_{+}(\cdot, k), F_{-}(\cdot, k)\right]} \text { and } R_{2}(x, y)=-\frac{G_{-}(x, k)^{t}\left(\sigma_{3} G_{+}(y, k)\right)}{W\left[G_{+}(\cdot, k), G_{-}(\cdot, k)\right]},
$$

and for $x>y$

$$
R_{1}(x, y)=-\frac{F_{+}(x, k)^{t}\left(\sigma_{3} F_{-}(y, k)\right)}{W\left[F_{+}(\cdot, k), F_{-}(\cdot, k)\right]} \text { and } R_{2}(x, y)=-\frac{G_{+}(x, k)^{t}\left(\sigma_{3} G_{-}(y, k)\right)}{W\left[G_{+}(\cdot, k), G_{-}(\cdot, k)\right]} .
$$

We have $R_{H_{\omega}}(x, y, \bar{\lambda})=\overline{R_{H_{\omega}}(x, y, \lambda)}$ and $R_{H_{\omega}}(x, y,-\lambda)=-\sigma_{1} R_{H_{\omega}}(x, y, \lambda) \sigma_{1}$.

We define:

Definition 8.11 (Plane waves). For $k \notin S$ set $\Psi(x, k):=\frac{1}{\sqrt{2 \pi}} T(k) F_{+}(x, k)$ for $k \geq$ 0 and $\Psi(x, k):=\frac{1}{\sqrt{2 \pi}} T(-k) F_{-}(x,-k)$ for $k<0$. Near $S$ set $\Psi(x, k)=\frac{1}{\sqrt{2 \pi}} \Phi_{+}(x, k)$ for $k>0$ and $\Psi(x, k)=\frac{1}{\sqrt{2 \pi}} \Phi_{-}(x,-k)$ for $k<0$.

The interpretation of the $\Psi(x, k)$ is justified by the following lemma:

Lemma 8.12. The operator $P_{c}\left(H_{\omega}\right)$ has, for ${ }^{t} A$ the transpose of $A$, the kernel

$$
P_{c}\left(H_{\omega}\right)(x, y)=\int_{\mathbb{R}}\left[\overline{\Psi(x, k)}^{t}\left(\sigma_{3} \Psi(y, k)\right)+\sigma_{1} \overline{\Psi(x, k)}^{t}\left(\sigma_{3} \sigma_{1} \Psi(y, k)\right)\right] d k .
$$

In particular we have $\left(e^{i t H_{\omega}} P_{c}\left(H_{\omega}\right)\right)(x, y)=$

$$
\int_{\mathbb{R}}\left[e^{i t(E(k)+\omega)} \overline{\Psi(x, k)}{ }^{t}\left(\sigma_{3} \Psi(y, k)\right)+e^{-i t(E(k)+\omega)} \sigma_{1} \overline{\Psi(x, k)}{ }^{t}\left(\sigma_{3} \sigma_{1} \Psi(y, k)\right)\right] d k .
$$


Lemma 8.13. The following operators $P_{ \pm}(\omega)$ are well defined in $L_{x}^{2}$ :

$$
\begin{aligned}
& P_{+}(\omega) u=\lim _{M \nearrow \infty \epsilon \searrow 0} \lim _{\epsilon} \frac{1}{2 \pi i} \int_{[\omega, M] \cap\left(\omega+\sigma\left(h_{0}\right)\right)}\left[R_{H_{\omega}}(\lambda+i \epsilon)-R_{H_{\omega}}(\lambda-i \epsilon)\right] u d \lambda,
\end{aligned}
$$

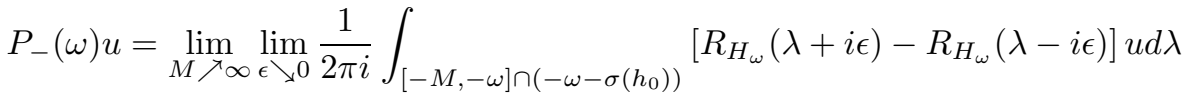

and have kernel

$$
\begin{aligned}
& P_{+}(\omega)(x, y)=\int_{\mathbb{R}} \overline{\Psi(x, k)}^{t}\left(\sigma_{3} \Psi(y, k)\right) d k, \\
& P_{-}(\omega)(x, y)=\int_{\mathbb{R}} \sigma_{1} \overline{\Psi(x, k)}^{t}\left(\sigma_{3} \sigma_{1} \Psi(y, k)\right) d k .
\end{aligned}
$$

For any $M>0$ and $N>0$ and for $C=C(N, M, \omega)$ upper semicontinuous in $\omega$, we have

$$
\left\|\langle x\rangle^{M}\left(P_{+}(\omega)-P_{-}(\omega)-P_{c}\left(H_{\omega}\right) \sigma_{3}\right) f\right\|_{L_{x}^{2}} \leq C\left\|\langle x\rangle^{-N} f\right\|_{L_{x}^{2}}
$$

\section{Proof of Lemmas 3.1-3.2}

Lemma 3.1 is a consequence of the following statement:

Lemma 9.1. Set $P_{c}\left(H_{\omega}\right) e^{i t H_{\omega}}(x, y)=\mathcal{U}(t, x, y)+\mathcal{V}(t, x, y)$ with

$$
\begin{aligned}
& \mathcal{U}(t, x, y)=\int_{|k| \leq \pi n\left(n_{0}\right)} e^{i t E(k)} \phi(x, y, k) d k, \\
& \mathcal{V}(t, x, y)=\int_{|k| \geq \pi n\left(n_{0}\right)} e^{i t E(k)} \phi(x, y, k) d k \\
& \text { with } \phi(x, y, k)=\overline{\Psi(x, k)}{ }^{t}\left(\sigma_{3} \Psi(y, k)\right)+\sigma_{1} \overline{\Psi(x, k)}{ }^{t}\left(\sigma_{3} \sigma_{1} \Psi(y, k)\right) .
\end{aligned}
$$

Then $|\mathcal{U}(t, x, y)| \leq C\langle t\rangle^{-\frac{1}{3}}$ and $|\mathcal{V}(t, x, y)| \leq C|t|^{-\frac{1}{2}}$.

Proof. Using cutoff functions we distinguish between $k$ close to $S$ and $k$ away from $S$. In the latter case the proof is the same as Lemma 6.5. So for $\chi(k)$ a smooth cutoff function with small support $I$ with $I \cap S=\{\widehat{k}\}$ and with $I \subset \mathbb{R}_{+}$(the above choices are not restrictive) contained either in $|k|<\pi n\left(n_{0}\right)$ or in $|k|>\pi n\left(n_{0}\right)$, we consider the integral

$$
\int_{I} e^{i t E(k)} \chi(k) \bar{\Phi}_{+}(x, k){ }^{t} \Phi_{-}(y, k) d k .
$$

Notice that by $(8.6)$

$$
\Phi_{+}(x, k)=\phi_{ \pm}^{0}(x, k) \vec{e}_{1}(1+A(x, k))+\phi_{-}^{0}(x, k) B(x, k)+C(x, y, k)
$$

with $\left|\partial_{k}^{a} A(x, k)\right|+\left|\partial_{k}^{a} B(x, k)\right|+\left|\partial_{k}^{a} C(x, y, k)\right|<c_{0}$ for fixed $c_{0}$ for $a=0,1$. Then by stationary phase $|(1)| \leq\langle t\rangle^{-\alpha}$ with $\alpha$ either $1 / 2$ if $I$ is in $|k|<\pi n\left(n_{0}\right)$ or $1 / 3$ if $I$ is in $|k|>\pi n\left(n_{0}\right)$. Before proving Lemma 3.2 we need:

Lemma 9.2. Let $I_{\alpha, \beta}[f]=K_{\alpha, \beta}(t) * f$ with $K_{\alpha, \beta} \equiv \chi_{[0,1)} t^{-\alpha}+\chi_{[1, \infty)} t^{-\beta}$ for given $0<\alpha<1$ and $0<\beta<1$. Then we have the following estimates:

$$
\left\|I_{\alpha, \beta}[f]\right\|_{\ell^{p}\left(\mathbb{Z}, L_{t}^{r}[n, n+1]\right)} \leq C\|f\|_{\ell^{q}\left(\mathbb{Z}, L_{t}^{s}[n, n+1]\right)},
$$


where $C \equiv C(p, q, r, s)>0$ and

$$
\begin{aligned}
& 1+\frac{1}{r} \geq \alpha+\frac{1}{s}, \\
& 1+\frac{1}{p} \leq \beta+\frac{1}{q}, \\
& (r, s) \neq\left(\infty, \frac{1}{1-\alpha}\right),\left(\frac{1}{\alpha}, 1\right), \\
& (p, q) \neq\left(\infty, \frac{1}{1-\beta}\right),\left(\frac{1}{\beta}, 1\right) .
\end{aligned}
$$

If moreover we assume $\alpha=0$ and $0<\beta<1$, then

$$
\left\|I_{0, \beta} f\right\|_{\ell^{p}\left(\mathbb{Z}, L_{t}^{r}[n, n+1]\right)} \leq C\|f\|_{\ell^{q}\left(\mathbb{Z}, L_{t}^{s}[n, n+1]\right)},
$$

where $1 \leq r, s \leq \infty$ and $1 \leq p, q \leq \infty$ satisfy (2) and (4).

The elementary proof is in Lemma 2.2 of $\mathrm{GV}$.

Proof of Lemma 3.2. Using the definition of $\mathcal{U}(t)$ and $\mathcal{V}(t)$, the proof of the Strichartz estimates for (2) and (4) of Lemma 3.2 are the same as that in Lemma 3.1 of [C1]. We turn now to the estimate for $\mathcal{U}(t)$. In the sequel the pairs $(r, p)$, $\left(r_{1}, p_{1}\right)$ and $\left(r_{2}, p_{2}\right)$ are always admissible. First of all, by $\left[H_{\omega}, \mathcal{U}(t)\right]=0$, it is not restrictive to consider only case $k=0$. We set $P_{ \pm}=P_{ \pm}\left(H_{\omega}\right)$. We have $\left[\mathcal{U}, P_{ \pm}\right]=0$, $\mathcal{U}^{*} \sigma_{3}=\sigma_{3} \mathcal{U}$ and $P_{ \pm}^{*} \sigma_{3}=\sigma_{3} P_{ \pm}$.

First step: Proof of (1) Lemma 3.2. The case $(r, p)=(\infty, 2)$ is trivial; hence by interpolation it suffices to prove $(1)$ in the case $(r, p)=(4, \infty)$. By Lemma 3.1

$$
\left\|\int_{0}^{\infty} \mathcal{U}(t-s) F(s) d s\right\|_{L_{x}^{\infty}} \leq C \int_{0}^{\infty}\|F(s)\|_{L_{x}^{1}} \frac{d s}{\langle t-s\rangle^{\frac{1}{3}}} .
$$

By Lemma 9.2 with $\alpha=0$ and $\beta=\frac{1}{3}$,

$$
\left\|\int_{0}^{\infty} \mathcal{U}(t-s) F(s) d s\right\|_{\ell^{6}\left(\mathbb{Z}, L_{t}^{\infty}\left([n, n+1], L_{x}^{\infty}\right)\right)} \leq C\|F\|_{\ell^{\frac{6}{5}\left(\mathbb{Z}, L_{t}^{1}\left([n, n+1], L_{x}^{1}\right)\right)}} .
$$

By Fubini's theorem we have

$$
\begin{aligned}
& \left\langle\int_{0}^{\infty} \mathcal{U}(s) P_{ \pm} F(s) d s, \sigma_{3} \int_{0}^{\infty} \mathcal{U}(t) P_{ \pm} F(t) d t\right\rangle_{x} \\
& =\left\langle\int_{0}^{\infty} \mathcal{U}(t-s) P_{ \pm} F(s) d s, \sigma_{3} P_{ \pm} F(t)\right\rangle_{t, x} .
\end{aligned}
$$

This implies

$$
\begin{aligned}
& \left\|\int_{0}^{\infty} \mathcal{U}(s) F(s) d s\right\|_{L_{x}^{2}}^{2} \lesssim\left\|\int_{0}^{\infty} \mathcal{U}(t-s) F(s) d s\right\|_{\ell^{6}\left(\mathbb{Z}, L_{t}^{\infty}\left([n, n+1], L_{x}^{\infty}\right)\right)} \\
& \times\|F\|_{\ell^{\frac{6}{5}}\left(\mathbb{Z}, L_{t}^{1}\left([n, n+1], L_{x}^{1}\right)\right)} \leq C\|F\|_{\ell^{\frac{6}{5}}\left(\mathbb{Z}, L_{t}^{1}\left([n, n+1], L_{x}^{1}\right)\right)}^{2}
\end{aligned}
$$

by (9.1) and by $\left\|P_{ \pm} u\right\|_{L_{x}^{2}}^{2} \approx\left|\left\langle P_{ \pm} u, \sigma_{3} P_{ \pm} u\right\rangle\right|$. The latter follows from the following facts, for $W$ and $Z$ the operators in Proposition 7.1: for $u \in L_{c}^{2}\left(H_{\omega}\right)$ and $u=W v$, we have $\left\|P_{+} u\right\|_{L_{x}^{2}} \approx\|\operatorname{diag}(1,0) v\|_{L_{x}^{2}},\left\|P_{-} u\right\|_{L_{x}^{2}} \approx\|\operatorname{diag}(0,1) v\|_{L_{x}^{2}}$; for any pair $\widetilde{u}=W \widetilde{v}$ we have $\left\langle\widetilde{u}, \sigma_{3} \widetilde{u}\right\rangle=\left\langle\widetilde{v}, \sigma_{3} \widetilde{v}\right\rangle$. 
To finally deduce (1) of Lemma 3.2, notice that by combining the Fubini theorem, a duality argument, the Hölder inequality and (9.2), we get for $f \in L_{c}^{2}\left(H_{\omega}\right)$

$$
\begin{aligned}
& \|\mathcal{U}(t) f\|_{\ell^{6}\left(\mathbb{Z}, L_{t}^{\infty}\left([n, n+1], L_{x}^{\infty}\right)\right)}=\sup _{G \in \mathcal{B}_{\frac{6}{5}, 1,1}}\left\langle\mathcal{U}(t) f, \sigma_{3} G(t, x)\right\rangle_{t, x} \\
& =\sup _{G \in \mathcal{B}_{\frac{6}{5}, 1,1}}\left\langle f, \sigma_{3} \int_{0}^{\infty} \mathcal{U}(t) P_{c}\left(H_{\omega}\right) G(t) d t\right\rangle_{x} \\
& \leq\|f\|_{L_{x}^{2}} \sup _{G \in \mathcal{B}_{\frac{6}{5}, 1,1}}\left\|\int_{0}^{\infty} \mathcal{U}(t) P_{c}\left(H_{\omega}\right) G(t) d t\right\|_{L_{x}^{2}} \leq C\|f\|_{L_{x}^{2}},
\end{aligned}
$$

where

$$
\mathcal{B}_{\frac{6}{5}, 1,1}:=\left\{G \in \ell^{\frac{6}{5}}\left(\mathbb{Z}, L_{t}^{1}\left([n, n+1], L_{x}^{1}\right)\right) \text { s.t. }\|G\|_{\ell^{\frac{6}{5}}\left(\mathbb{Z}, L_{t}^{1}\left([n, n+1], L_{x}^{1}\right)\right)}=1\right\} .
$$

Second step: Proof of (3) Lemma 3.2. We split the proof in 7 subcases.

First subcase: $\left(r_{i}, p_{i}\right)=(\infty, 2)$ for $i=1,2$. In this case the estimate (3) of Lemma 3.2 is equivalent to the following one:

$$
\left\|\int_{0}^{t} \mathcal{U}(t-s) F(s) d s\right\|_{L_{t}^{\infty} L_{x}^{2}} \leq C\|F\|_{L_{t}^{1} L_{x}^{2}}
$$

whose proof is elementary,

$$
\left\|\int_{0}^{t} \mathcal{U}(t-s) F(s) d s\right\|_{L_{x}^{2}} \leq \int_{0}^{t}\|\mathcal{U}(t-s) F(s)\|_{L_{x}^{2}} d s \leq C \int_{0}^{t}\|F(s)\|_{L_{x}^{2}} d s,
$$

where we have used the fact that $\|\mathcal{U}(t) f\|_{L_{x}^{2}} \leq C\|f\|_{L_{x}^{2}}$ for a fixed $C$.

Second subcase: $\left(r_{i}, p_{i}\right)=(4, \infty)$ for $i=1,2$. It is similar to the proof of $(9.1)$.

Third subcase: $\left(r_{1}, p_{1}\right)=(4, \infty)$ and $\left(r_{2}, p_{2}\right)=(\infty, 2)$. In this case (3) of Lemma 3.2 reduces to

$$
\left\|\int_{0}^{t} \mathcal{U}(t-s) F(s) d s\right\|_{\ell^{6}\left(\mathbb{Z}, L_{t}^{\infty}\left([n, n+1], L_{x}^{\infty}\right)\right)} \leq C\|F\|_{L_{t}^{1} L_{x}^{2}} .
$$

Notice that we have the identity

$$
\int_{0}^{t} \mathcal{U}(t-s) F(s) d s=\int_{0}^{\infty} \chi(t-s) \mathcal{U}(t-s) F(s) d s
$$

with $\chi$ the characteristic function of the half line $(0, \infty)$. By the Minkowski inequality we get

$$
\begin{gathered}
\left\|\int_{0}^{t} \mathcal{U}(t-s) F(s) d s\right\|_{\ell^{6}\left(\mathbb{Z}, L_{t}^{\infty}\left([n, n+1], L_{x}^{\infty}\right)\right)} \\
\leq \int_{0}^{\infty}\|\chi(t-s) \mathcal{U}(t-s) F(s)\|_{\ell^{6}\left(\mathbb{Z}, L_{t}^{\infty}\left([n, n+1], L_{x}^{\infty}\right)\right)} d s \\
\leq \int_{0}^{\infty}\|\mathcal{U}(t-s) F(s)\|_{\ell^{6}\left(\mathbb{Z}, L_{t}^{\infty}\left([n, n+1], L_{x}^{\infty}\right)\right)} d s \leq C \int_{0}^{\infty}\|F(s)\|_{L_{x}^{2}} d s,
\end{gathered}
$$

where we have used (1) of Lemma 3.2 in the last step.

Fourth subcase: $\left(r_{1}, p_{1}\right)$ any admissible pair and $\left(r_{2}, p_{2}\right)=(\infty, 2)$. This follows by interpolation between the first and the third subcases. 
Fifth subcase: $\left(r_{1}, p_{1}\right)=(\infty, 2),\left(r_{2}, p_{2}\right)=(4, \infty)$. This follows by a duality argument. In fact we have

$$
\begin{gathered}
\left\|\int_{0}^{t} \mathcal{U}(t-s) F(s) d s\right\|_{L_{t}^{\infty} L_{x}^{2}}=\sup _{G \in \mathcal{B}_{1,2}}\left\langle\int_{0}^{t} \mathcal{U}(t-s) F(s) d s, \sigma_{3} G\right\rangle_{t, x} \\
=\sup _{G \in \mathcal{B}_{1,2}}\left\langle F, \sigma_{3} \int_{t}^{\infty} \mathcal{U}(t-s) G(s) d s\right\rangle_{t, x},
\end{gathered}
$$

where

$$
\mathcal{B}_{1,2}:=\left\{G \in L_{t}^{1} L_{x}^{2} \text { s.t. }\|G\|_{L_{t}^{1} L_{x}^{2}}=1\right\} .
$$

By Hölder inequality

$$
\begin{aligned}
& \left\|\int_{0}^{t} \mathcal{U}(t-s) F(s) d s\right\|_{L_{t}^{\infty} L_{x}^{2}} \leq\|F\|_{\ell^{\frac{6}{5}}\left(\mathbb{Z}, L_{t}^{1}\left([n, n+1], L_{x}^{1}\right)\right)} \\
& \times \sup _{G \in \mathcal{B}_{1,2}}\left\|\int_{t}^{\infty} \mathcal{U}(t-s) G(s) d s\right\|_{\ell^{6}\left(\mathbb{Z}, L_{t}^{\infty}\left([n, n+1], L_{x}^{\infty}\right)\right)} \leq C\|F\|_{\ell^{\frac{6}{5}}\left(\mathbb{Z}, L_{t}^{1}\left([n, n+1], L_{x}^{1}\right)\right)},
\end{aligned}
$$

where in the last step we used the estimate

$$
\left\|\int_{t}^{\infty} \mathcal{U}(t-s) G(s) d s\right\|_{\ell^{6}\left(\mathbb{Z}, L_{t}^{\infty}\left([n, n+1], L_{x}^{\infty}\right)\right)} \leq C\|G\|_{L_{t}^{1} L_{x}^{2}},
$$

whose proof is similar to (9.3).

Sixth subcase: $\left(r_{1}, p_{1}\right)$ any admissible pair and $\left(r_{2}, p_{2}\right)=(4, \infty)$. This follows by interpolation of the second and fifth cases.

Seventh subcase: Remaining cases. This follows by interpolation of the fourth and sixth cases.

\section{Extension of a Result by Christ and Kiselev TO BIRMAN-SOLOMJAK SPACES}

Given two Banach spaces $X$ and $Y$ let $K(s, t)$ be a continuous function with values in $B(X, Y)$. Let us introduce the operators

$$
T_{K} f(t)=\int_{-\infty}^{\infty} K(t, s) f(s) d s
$$

and

$$
\tilde{T}_{K} f(t)=\int_{-\infty}^{t} K(t, s) f(s) d s .
$$

In this section we shall prove the following modified version of Lemma $3.1 \mathrm{in}$ [SmS].

Proposition 10.1. Let $1 \leq p, q, r \leq \infty$ be such that $1 \leq r<\min \{p, q\} \leq \infty$. Assume that there exist $C>0$ such that

$$
\left\|T_{K} f\right\|_{\ell^{q}\left(\mathbb{Z}, L_{t}^{p}([n, n+1], Y)\right)} \leq C\|f\|_{L_{t}^{r}(X)} .
$$

Then

$$
\left\|\tilde{T}_{K} f\right\|_{\ell^{q}\left(\mathbb{Z}, L_{t}^{p}([n, n+1], Y)\right)} \leq C^{\prime}\|f\|_{L_{t}^{r}(X)},
$$

where $C^{\prime}=C^{\prime}(C, p, q, r)>0$ is another suitable constant.

Remark. In the case $p=q$ the previous proposition follows from [CK].

We shall need the following lemma. 
Lemma 10.2. Let $k \in \mathbb{N}$ and $1 \leq p, q, r \leq \infty$ be as in Proposition 10.1. Assume that $\left\{I_{j}\right\}_{j=1, \ldots, 2^{k}} \subset \mathbb{R}$ is a family of intervals (eventually unbounded) such that

$$
\mathbb{R}=\bigcup_{j} I_{j} \text { and } i n t I_{j} \cap i n t I_{k}=\emptyset \text { for } j \neq k .
$$

Assume also that $g_{j} \in \ell^{q}\left(\mathbb{Z}, L_{t}^{p}([n, n+1], Y)\right)$ for $j=1, \ldots, 2^{k}$ is a family of functions such that

$$
\left\|g_{j}\right\|_{\ell q\left(\mathbb{Z}, L_{t}^{p}([n, n+1], Y)\right)} \leq C 2^{-\frac{k}{r}} \forall j=1, \ldots, 2^{k} .
$$

Then the following estimate holds:

$$
\left\|\sum_{j=1}^{2^{k}} \chi_{I_{j}} g_{j}\right\|_{\ell^{q}\left(\mathbb{Z}, L_{t}^{p}([n, n+1], Y)\right)} \leq C^{\prime} 2^{k\left(\frac{1}{q}-\frac{M i n\{p, q\}}{q r}\right)},
$$

where $C^{\prime}=C^{\prime}(C, p, q, r)>0$ and in particular $C^{\prime}$ does not depend on $g_{j}, I_{j}, k$.

Proof. Let us fix the following notation:

$$
G(x) \equiv \sum_{j=1}^{2^{k}} \chi_{I_{j}} g_{j}
$$

and

$$
\mathcal{I} \equiv\left\{I_{j} \text { for } j=0,1, \ldots, 2^{k}\right\},
$$

where $I_{j}$ are the intervals given in the statement and $I_{0} \equiv \emptyset$. To every $I_{j} \in \mathcal{I}$ we associate a new segment $\tilde{I}_{j} \subset I_{j}$ defined as follows:

$$
\tilde{I}_{j} \equiv\left(z_{j}, z_{j+1}\right),
$$

where $z_{j}, z_{j+1} \in \mathbb{Z} \cup\{ \pm \infty\}, z_{j} \equiv \inf \left\{z \in \mathbb{Z} \cup\{-\infty\} \mid z \in I_{j}\right\}$ and $z_{j+1} \equiv$ $\sup \left\{z \in \mathbb{Z} \cup\{+\infty\} \mid z \in I_{j}\right\}$. Notice that the possibility $\tilde{I}_{j}=\emptyset$ is allowed. Next we introduce

$$
\tilde{\mathcal{I}} \equiv\left\{I_{j} \in \mathcal{I} \mid \tilde{I}_{j} \neq \emptyset\right\}
$$

and also

$$
\tilde{\mathbb{Z}} \equiv\left\{z \in \mathbb{Z} \mid(z, z+1) \cap\left(\bigcup_{j=1}^{2^{k}} \tilde{I}_{j}\right)=\emptyset\right\} .
$$

Notice that in a more explicit way we can write

$$
\tilde{\mathcal{I}}=\left\{I_{i_{0}}, I_{i_{1}}, \ldots, I_{i_{h}}\right\}
$$

for suitable $0 \leq h \leq 2^{k}$ and $0=i_{0}<i_{1}<\ldots<i_{h} \leq 2^{k}$. For every function $F \in l^{q}\left(\mathbb{Z}, L_{t}^{p}([n, n+1], Y)\right)$ we get

$$
\left\|\chi_{\left(\bigcup_{I_{j} \in \tilde{I}} \tilde{I}_{j}\right)} F\right\|_{\ell^{q}\left(\mathbb{Z}, L_{t}^{p}([n, n+1], Y)\right)}=\left(\sum_{j=i_{0}, \ldots, i_{h}}\left\|\chi_{\tilde{I}_{j}} F\right\|_{\ell^{q}\left(\mathbb{Z}, L_{t}^{p}([n, n+1], Y)\right)}^{q}\right)^{\frac{1}{q}} .
$$


In particular, if we choose in this identity $F=G$, with $G$ as in (10.5), then we get

$$
\begin{aligned}
& \left\|\chi_{\left(\cup_{I_{j} \in \tilde{I}} \tilde{I}_{j}\right)} G\right\|_{\ell^{q}\left(\mathbb{Z}, L_{t}^{p}([n, n+1], Y)\right)}^{q}=\left\|\chi_{\left(\cup_{I_{j} \in \tilde{I}} \tilde{I}_{j}\right)}\left(\sum_{l=1}^{2^{k}} \chi_{I_{l}} g_{l}\right)\right\|_{\ell^{q}\left(\mathbb{Z}, L_{t}^{p}([n, n+1], Y)\right)}^{q} \\
& \quad=\sum_{j=i_{0}, i_{1}, \ldots, i_{h}}\left\|\chi_{\tilde{I}_{j}} g_{j}\right\|_{\ell^{q}\left(\mathbb{Z}, L_{t}^{p}([n, n+1], Y)\right)}^{q}\left\|\sum_{j=i_{0}, i_{1}, \ldots, i_{h}}\right\| g_{j} \|_{\ell^{q}\left(\mathbb{Z}, L_{t}^{p}([n, n+1], Y)\right)}^{q} \leq C^{q} h 2^{-\frac{k q}{r}},
\end{aligned}
$$

where we have used the assumption (10.3). Next we shall estimate

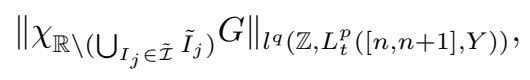

with $G$ as in (10.5). In order to do that let us associate to any integer $n_{0} \in \tilde{\mathbb{Z}}$, defined in (10.6), the following set:

$$
\mathcal{I}_{n_{0}} \equiv\left\{I \in \mathcal{I} \mid I \cap\left(n_{0}, n_{0}+1\right) \neq \emptyset\right\} .
$$

In particular, we can write in a more explicit way:

$$
\mathcal{I}_{n_{0}} \equiv\left\{I_{i\left(n_{0}\right)}, I_{i\left(n_{0}\right)+1}, \ldots, I_{i\left(n_{0}\right)+h\left(n_{0}\right)}\right\},
$$

where

$$
0 \leq h\left(n_{0}\right) \leq 2^{k}, i\left(n_{0}\right) \in 1, \ldots, 2^{k} .
$$

It is easy to deduce that the assumptions on $\left\{I_{j}\right\}_{j=1, . .2^{k}}$ imply

$$
0 \leq h \leq 2^{k} \text { and } \sum_{n_{0} \in \tilde{\mathbb{Z}}} h\left(n_{0}\right) \leq 2^{k+1} .
$$

Next notice that for every $n_{0} \in \tilde{\mathbb{Z}}$ we have

$$
\begin{aligned}
& \left\|\chi_{\left(n_{0}, n_{0}+1\right)} G\right\|_{L^{p}\left(\left[n_{0}, n_{0}+1\right], Y\right)}^{p}=\sum_{J \in \mathcal{I}_{n_{0}}}\left\|\chi_{J \cap\left[n_{0}, n_{0}+1\right]} G\right\|_{L^{p}\left(\left[n_{0}, n_{0}+1\right], Y\right)}^{p} \\
& =\sum_{h=0}^{h\left(n_{0}\right)}\left\|\chi_{I_{i\left(n_{0}\right)+h} \cap\left[n_{0}, n_{0}+1\right]} g_{i\left(n_{0}\right)+h}\right\|_{L^{p}\left(\left[n_{0}, n_{0}+1\right], Y\right)}^{p} \\
& \leq \sum_{h=0}^{h\left(n_{0}\right)}\left\|g_{i\left(n_{0}\right)+h}\right\|_{L^{p}\left(\left[n_{0}, n_{0}+1\right], Y\right)}^{p} \leq C^{p}\left(h\left(n_{0}\right)+1\right) 2^{-\frac{k p}{r}}
\end{aligned}
$$

and this implies

$$
\left\|\chi_{\left(n_{0}, n_{0}+1\right)} G\right\|_{L^{p}\left(\left[n_{0}, n_{0}+1\right], Y\right)}^{q} \leq C^{q} 2^{\frac{q}{p}} 2^{-\frac{k q}{r}} h\left(n_{0}\right)^{\frac{q}{p}}=C^{q} 2^{\frac{q}{p}}\left(2^{-\frac{k p}{r}} h\left(n_{0}\right)\right)^{\frac{q}{p}} .
$$

Next we split the proof of (10.4) into two cases.

First case: $q \geq p$

In this case we have the following estimate:

$$
C^{q} 2^{\frac{q}{p}}\left(2^{-\frac{k p}{r}} h\left(n_{0}\right)\right)^{\frac{q}{p}} \leq C^{q} 2^{\frac{q}{p}} 2^{-\frac{k p}{r}} h\left(n_{0}\right),
$$

where at the last step we have used the assumption $q \geq p$ and the following fact:

$$
2^{-\frac{k p}{r}} h\left(n_{0}\right) \leq 2^{-k} h\left(n_{0}\right) \leq 1,
$$

that in turn follows from the hypothesis $p>r$ and $0 \leq h\left(n_{0}\right) \leq 2^{k}$. 
By combining (10.7) with (10.9) and (10.10) we get

$$
\begin{aligned}
& \|G\|_{\ell^{q}\left(\mathbb{Z}, L_{t}^{p}([n, n+1], Y)\right)}^{q}=\left\|\chi_{\left(\cup_{I_{j} \in \tilde{I}} \tilde{I}_{j}\right)} G\right\|_{\ell^{q}\left(\mathbb{Z}, L_{t}^{p}([n, n+1], Y)\right)}^{q}+\sum_{n_{0} \in \tilde{\mathbb{R}}}\|G\|_{L^{p}\left(\left[n_{0}, n_{0}+1\right], Y\right)}^{q} \\
& \leq C^{q} 2^{-\frac{k q}{r}} h+\sum_{n_{0} \in \tilde{\mathbb{Z}}} C^{q} 2^{\frac{q}{p}} 2^{-\frac{k p}{r}} h\left(n_{0}\right),
\end{aligned}
$$

that due to (10.8) implies

$$
\|G\|_{\ell^{q}\left(\mathbb{Z}, L_{t}^{p}([n, n+1], Y)\right)}^{q} \leq C^{q} 2^{k\left(1-\frac{q}{r}\right)}+2 C^{q} 2^{\frac{q}{p}} 2^{k\left(1-\frac{p}{r}\right)} .
$$

Hence, due to the hypothesis $q \geq p>r$, we get

$$
\|G\|_{\ell^{q}\left(\mathbb{Z}, L_{t}^{p}([n, n+1], Y)\right)}^{q} \leq 3 C^{q} 2^{\frac{q}{p}} 2^{k\left(1-\frac{p}{r}\right)} .
$$

Second case: $q<p$

In this case we have

$$
C^{q} 2^{\frac{q}{p}}\left(2^{-\frac{k p}{r}} h\left(n_{0}\right)\right)^{\frac{q}{p}}=C^{q} 2^{\frac{q}{p}} 2^{-\frac{k q}{r}} h\left(n_{0}\right)^{\frac{q}{p}} \leq C^{q} 2^{\frac{q}{p}} 2^{-\frac{k q}{r}} h\left(n_{0}\right),
$$

where at the last step we have used $h\left(n_{0}\right) \geq 1$ and $q<p$. By combining (10.7) with (10.9) and (10.11) we get

$$
\begin{aligned}
& \|G\|_{\ell^{q}\left(\mathbb{Z}, L_{t}^{p}([n, n+1], Y)\right)}^{q}=\left\|\chi_{\left(\bigcup_{I_{j} \in \tilde{I}} \tilde{I}_{j}\right)} G\right\|_{\ell^{q}\left(\mathbb{Z}, L_{t}^{p}([n, n+1], Y)\right)}^{q}+\sum_{n_{0} \in \tilde{\mathbb{Z}}}\|G\|_{L^{p}\left(\left[n_{0}, n_{0}+1\right], Y\right)}^{q} \\
& \leq C^{q} 2^{-\frac{k q}{r}} h+\sum_{n_{0} \in \tilde{\mathbb{Z}}} C^{q} 2^{\frac{q}{p}} 2^{-\frac{k q}{r}} h\left(n_{0}\right) \leq 3 C^{q} 2^{\frac{q}{p}} 2^{k\left(1-\frac{q}{r}\right)}
\end{aligned}
$$

where at the last step we have used (10.8).

Proof of Proposition 10.1. We shall follow the notation used in SmS. Let $f \in$ $L_{t}^{r}(X)$ satisfy the following conditions:

(1) $\|f\|_{L_{t}^{r}(X)}=1$

(2) the function $F(t)$ defined below is a bijection between $(-\infty, \infty)$ and $(0,1)$,

$$
F(t)=\int_{-\infty}^{t}\|f(s)\|_{X}^{r} d s
$$

By an elementary density argument we have that (10.2) will follow once we show that $\left\|\tilde{T}_{K} f\right\|_{\ell^{q}\left(\mathbb{Z}, L_{t}^{p}([n, n+1], Y)\right)} \leq C^{\prime}$ for any $f \in L_{t}^{r}(X)$ that satisfies the conditions above. Next we consider the set of all dyadic subintervals of $[0,1]$. If $I$ and $J$ are two such subintervals, we say that $I \sim J$ if the following conditions hold:

(1) $I$ and $J$ have the same length;

(2) $I$ must lie on the left of $J$;

(3) there exist two dyadic cubes $I_{0}$ and $J_{0}$ whose length is twice the lenght of $I$ and $J$, and moreover $I_{0}$ and $J_{0}$ are adjacent.

Notice that if $J$ is fixed, then there are two dyadic intervals such that $I \sim J$. Following $\mathrm{CK}$, $\mathrm{SmS}$, we can write the identity

$$
\tilde{T}_{K} f=\sum_{\{I, J: I \sim J\}} \chi_{F^{-1} J} T_{K}\left(\chi_{F^{-1} I} f\right)
$$


where $\chi_{A}$ denotes the characteristic function of the set $A$ and $F(t)$ is given in (10.12). Due to the Minkowski inequality we get

$$
\begin{aligned}
& \left\|\tilde{T}_{K} f\right\|_{\ell^{q}\left(\mathbb{Z}, L_{t}^{p}([n, n+1], Y)\right)} \\
& \leq \sum_{k=2}^{\infty}\left\|\sum_{\left\{I, J: I \sim J,|I|=2^{-k}\right\}} \chi_{F^{-1} J} T\left(\chi_{F^{-1} I} f\right)\right\|_{\ell^{q}\left(\mathbb{Z}, L_{t}^{p}([n, n+1], Y)\right) .}
\end{aligned}
$$

Next for every $k \geq 2$ we fix the following notation:

$$
\begin{gathered}
I_{j}^{k} \equiv F^{-1}\left(\frac{j}{2^{k}}, \frac{j+1}{2^{k}}\right) \text { for } j=0,1, \ldots, 2^{k}-1, \\
f_{j}^{k}=\chi_{I_{j}^{k}} f \text { and } g_{j}^{k}=T_{K}\left(f_{j}^{k}\right) .
\end{gathered}
$$

Notice that

$$
\left\|f_{j}^{k}\right\|_{L_{t}^{r}(X)}=2^{-\frac{k}{r}} \quad \forall j=0,1, \ldots, 2^{k}-1,
$$

and due to the assumption (10.2) we deduce that

$$
\begin{aligned}
& \left\|g_{j}^{k}\right\|_{\ell^{q}\left(\mathbb{Z}, L_{t}^{p}([n, n+1], Y)\right)} \\
& =\left\|T_{K}\left(f_{j}^{k}\right)\right\|_{\ell^{q}\left(\mathbb{Z}, L_{t}^{p}([n, n+1], Y)\right)} \leq C 2^{-\frac{k}{r}} \quad \forall j=0, \ldots, 2^{k}-1 .
\end{aligned}
$$

We are then in a position to use Lemma 10.2 in order to deduce

$$
\begin{aligned}
& \left\|\sum_{\left\{I, J: I \sim J,|I|=2^{-k}\right\}} \chi_{F^{-1} J} T_{K}\left(\chi_{F^{-1} I} f\right)\right\|_{\ell^{q}\left(\mathbb{Z}, L_{t}^{p}([n, n+1], Y)\right)} \\
& =\left\|\sum_{j=2, \ldots, 2^{k}-1} \chi_{I_{j-1}^{k}} g_{j}^{k}(x)+\sum_{j=2, \ldots, 2^{k}-1} \chi_{I_{j-2}^{k}} g_{j}^{k}(x)\right\|_{\ell^{q}\left(\mathbb{Z}, L_{t}^{p}([n, n+1], Y)\right)} \\
& \leq\left\|\sum_{j=2, \ldots, 2^{k}} \chi_{I_{j-1}^{k}} g_{j}^{k}(x)\right\|_{\ell^{q}\left(\mathbb{Z}, L_{t}^{p}([n, n+1], Y)\right)}+\left\|\sum_{j=2, \ldots, 2^{k}} \chi_{I_{j-2}^{k}} g_{j}^{k}(x)\right\|_{\ell q}\left(\mathbb{Z}, L_{t}^{p}([n, n+1], Y)\right) \\
& \leq 2 C^{\prime} 2^{k\left(\frac{1}{q}-\frac{M i n\{p, q\}}{q r}\right)} .
\end{aligned}
$$

By (10.13) we finally get

$$
\left\|\tilde{T}_{K} f\right\|_{\ell^{q}\left(\mathbb{Z}, L_{t}^{p}([n, n+1], Y)\right)} \leq 2 C^{\prime} \sum_{k=2}^{\infty} 2^{k\left(\frac{1}{q}-\frac{\operatorname{Min}\{p, q\}}{q r}\right)},
$$

and since $\frac{1}{q}-\frac{\operatorname{Min}\{p, q\}}{q r}<0$ we have the desired result.

\section{REFERENCES}

[BP1] V.S.Buslaev, G.S.Perelman, Scattering for the nonlinear Schrödinger equation: states close to a soliton, St. Petersburg Math. J. 4 (1993), 1111-1142. MR.1199635 (94b:35256)

[BP2] - On the stability of solitary waves for nonlinear Schrödinger equations, Nonlinear evolution equations (N.N. Uraltseva, ed.), Transl. Ser. 2, 164, Amer. Math. Soc., Providence, RI, 1995, pp. 75-98. MR1334139 (96e:35157)

[BR] J.Bronski, Z.Rapti, Modulation instability for nonlinear Schrödinger equations with a periodic potential, Dy. Partial Diff. Equ. 2 (2005), 335-355. MR2193631 (2006k:35270)

[BS] V.S.Buslaev, C.Sulem, On the asymptotic stability of solitary waves of nonlinear Schrödinger equations, Ann. Inst. H. Poincaré. An. Nonlin. 20 (2003), 419-475. MR.1972870 (2004d:35229)

[Ca] T.Cazenave, Semilinear Schrödinger equations, Courant Lecture Notes, Amer. Math. Soc., 2003. MR2002047 (2004j:35266)

[CK] M.Christ, A.Kiselev, Maximal functions associated with filtrations, J. Funct. Anal. 179 (2001), 409-425. MR.1809116 (2001i:47054) 
[C1] S.Cuccagna, A revision of "On asymptotic stability in energy space of ground states of NLS in 1D", http://arxiv.org/abs/0711.4192.

[C2] Dispersion for Schrödinger equation with periodic potential in $1 D$, Communications PDE 33 (2008), 2064-2095. MR2475330

[C3] _ Stability of standing waves for NLS with perturbed Lamé potential, J. Differential Equations 223 (2006), 112-160. MR2210141 (2007e:35256)

[C4] Stabilization of solutions to nonlinear Schrödinger equations, Comm. Pure App. Math. 54 (2001), 1110-1145. MR.1835384(2002g:35193)

[C5] - On asymptotic stability of ground states of NLS, Rev. Math. Phys. 15 (2003), 877-903. MR2027616 (2004k:35348)

[C6] - On instability of excited states of the nonlinear Schrödinger equation, Physica D 238 (2009), 38-54. MR2571965

[C7] - On asymptotic stability in energy space of ground states of NLS in $1 D$, J. Differential Equations 245 (2008), 653-691. MR2422523 (2009c:35435)

[CM] S.Cuccagna, T.Mizumachi, On asymptotic stability in energy space of ground states for Nonlinear Schrödinger equations, http://arxiv.org/.

[CT] S.Cuccagna, M.Tarulli, On asymptotic stability in energy space of ground states of NLS in $2 D$, Ann. Inst. H. Poincaré Anal. Non Linéaire 26 (2009), no. 4, 1361-1386. MR2542729

[CPV] S.Cuccagna, D.Pelinovsky, V.Vougalter, Spectra of positive and negative energies in the linearization of the NLS problem, Comm. Pure Appl. Math. 58 (2005), 1-29. MR2094265 (2005k:35374)

[CV1] S.Cuccagna, N.Visciglia, Scattering for small energy solutions of NLS with periodic potential in $1 D$, C. R. Math. Acad. Sci. Paris 347 (2009), 243-247. MR2537530

[CV2] _ On asymptotic stability of ground states of NLS with a finite bands periodic potential in $1 D$, Rev. Math. Phys. 15 (2003), no. 8, 877-903. MR2027616 (2004k:35348)

[DT] P.Deift, E.Trubowitz, Inverse scattering on the line, Comm. Pure Appl. Math. 32 (1979), 121-251. MR512420 (80e:34011)

[FK] S.Fassari, M.Klaus, Coupling constant thresholds of perturbed periodic Hamiltonians, J. Math. Phys. 39 (1998), 4369-4416. MR.1643257 (99j:81040)

[F1] N.Firsova, On the time decay of a wave packet in a one-dimensional finite band periodic lattice, J. Math. Phys. 37 (1996), 1171-1181. MR1377626 (97d:81036)

[F2] $\quad$ A direct and inverse scattering problem for a one-dimensional perturbed Hill operator, Math. USSR-Sb. 58 (1987), 351-388. MR865766 (88d:34028)

[GH] T.Gallay, M.Haragus, Stability of small periodic waves for the nonlinear Schrödinger equation, J. Diff. Equations 234 (2007), 544-581. MR2300667 (2007k:35457)

[GV] J.Ginibre, G.Velo, Time decay of finite energy solutions of the nonlinear Klein Gordon and Schrödinger equations, Annales Inst. H. Poincaré A 43 (1985), 399-442. MR824083 (87g:35208)

[GNT] S.Gustafson, K.Nakanishi, T.P.Tsai, Asymptotic Stability and Completeness in the Energy Space for Nonlinear Schrödinger Equations with Small Solitary Waves, Int. Math. Res. Notices 66 (2004), 3559-3584. MR2101699 (2005g:35268)

[GS1] Zhou Gang, I.M.Sigal, Asymptotic stability of nonlinear Schrödinger equations with potential, Rev. Math. Phys. 17 (2005), 1143-1207. MR2187292 (2006j:35220)

[GS2] _ Relaxation of Solitons in Nonlinear Schrödinger Equations with Potential, Advances in Math. 216 (2007), 443-490. MR2351368 (2008k:35441)

[GSS] M.Grillakis, J.Shatah, W.Strauss, Stability of solitary waves in the presence of symmetries, I, Jour. Funct. An. 74 (1987), 160-197. MR901236 (88g:35169)

$[\mathrm{KM}]$ E.Kirr, Ö. Mizrak, On the asymptotic stability of bound states in $3 D$ cubic Scrödinger equation including subcritical cases, http://arxiv.org/abs/0803.3377.

[KS] J. Krieger, W. Schlag, Stable manifolds for all monic supercritical focusing nonlinear Schrödinger equations in one dimension, J. Amer. Math. Soc. 19 (2006), 815-920. MR2219305 (2007b:35301)

[KZ1] E.Kirr, A.Zarnescu, On the asymptotic stability of bound states in $2 D$ cubic Scrödinger equation, Comm. Math. Phys. 272 (2007), 443-468. MR2300249(2008a:35266)

[KZ2] - On the asymptotic stability of bound states in $2 D$ cubic Scrödinger equation including subcritical cases, http://arxiv.org/abs/0805.3888.

[K] E.Korotyaev, The propagation of the waves in periodic media at large time, Math. Asymptot. Anal. 15 (1997), 1-24. MR1474809 (98h:34149) 
[M1] T.Mizumachi, Asymptotic stability of small solitons to $1 D$ NLS with potential, Jour. of Math. Kyoto University 48 (2008), 471-497. MR2511047 (2010h:35377)

[M2] _ Asymptotic stability of small solitons for $2 D$ Nonlinear Schrödinger equations with potential, Jour. of Math. Kyoto University 43 (2007), 599-620. MR2402517 (2010a:35247)

[P] G.Perelman, Asymptotic stability of solitons for nonlinear Schrödinger equations, Comm. in PDE 29 (2004), 1051-1095. MR2097576 (2005g:35277)

[PW] C.A.Pillet, C.E.Wayne, Invariant manifolds for a class of dispersive, Hamiltonian partial differential equations, J. Diff. Eq. 141 (1997), 310-326. MR1488355 (99b:35193)

[RSS] I.Rodnianski, W.Schlag, A.Soffer, Asymptotic stability of $N$-soliton states of NLS, preprint, 2003, http://arxiv.org/abs/math.AP/0309114.

[ShS] J.Shatah, W.Strauss, Instability of nonlinear bound states, Comm. Math. Phys. 100 (1985), 173-190. MR804458 (87b:35159)

[SmS] H.F.Smith, C.D.Sogge, Global Strichartz estimates for nontrapping perturbations of the Laplacian, Comm. Partial Differential Equations 25 (2000), 2171-2183. MR1789924 (2001j:35180)

[SW1] A.Soffer, M.Weinstein, Multichannel nonlinear scattering for nonintegrable equations, Comm. Math. Phys. 133 (1990), 116-146. MR.1071238(91h:35303)

[SW2] _ Multichannel nonlinear scattering II. The case of anisotropic potentials and data, J. Diff. Eq. 98 (1992), 376-390. MR.1170476 (93i:35137)

[SW3] _ Resonances, radiation damping and instability in Hamiltonian nonlinear wave equations, Invent. Math. 136 (1999), 9-74. MR1681113 (2000k:37119)

[SW4] Selection of the ground state for nonlinear Schrödinger equations, Rev. Math. Phys. 16 (2004), 977-1071. MR.2101776 (2005g:81095)

[SK] A. Stefanov, P.G.Kevrekidis, Asymptotic behaviour of small solutions for the discrete nonlinear Schrödinger and Klein-Gordon equations, Nonlinearity 18 (2005), 1841-1857. MR:2150357 (2006b:37158)

[Str] W.Strauss, Nonlinear wave equations, CBMS Regional Conf. Ser. Mat. 76, AMS, 1989, pp. 173-190. MR.1032250 (91g:35002)

[TY1] T.P.Tsai, H.T.Yau, Asymptotic dynamics of nonlinear Schrödinger equations: Resonance dominated and radiation dominated solutions, Comm. Pure Appl. Math. 55 (2002), 153216. MR 1865414 (2002i:35182)

[TY2] - Relaxation of excited states in nonlinear Schrödinger equations, Int. Math. Res. Not. 31 (2002), 1629-1673. MR.1916427 (2004i:35292)

[TY3] _ Classification of asymptotic profiles for nonlinear Schrödinger equations with small initial data, Adv. Theor. Math. Phys. 6 (2002), 107-139. MR1992875 (2004m:35254)

[Wd1] R. Weder, Center manifold for nonintegrable nonlinear Schrödinger equations on the line, Comm. Math. Phys. 170 (2000), 343-356. MR1799850 (2001m:37168)

$[\mathrm{Wd} 2] \_, L^{p} \rightarrow L^{\dot{p}}$ estimates for the Schrödinger equation on the line and inverse scattering for the nonlinear Schrödinger equation with a potential, J. Funct. Anal. 170 (2000), 37-68. MR:1736195 (2001e:35135)

[We1] M.Weinstein, Lyapunov stability of ground states of nonlinear dispersive equations, Comm. Pure Appl. Math. 39 (1986), 51-68. MR820338 (87f:35023)

[We2] _ Modulation stability of ground states of nonlinear Schrödinger equations, Siam J. Math. Anal. 16 (1985), 472-491. MR783974 (86i:35130)

Dismi, University of Modena and Reggio Emilia, via Amendola 2, Padiglione Morselli, REgGio EMilia 42100 ItALy

E-mail address: cuccagna.scipio@unimore.it

Dipartimento di Matematica "L. Tonelli", University of Pisa, Largo B. Pontecorvo 5, 56127 Pisa, ITALY

E-mail address: viscigli@dm.unipi.it 